\title{
Carrier-Free, Dual-Functional Nanorods Via Self-Assembly Of Pure Drug Molecules For Synergistic Chemo-Photodynamic Therapy
}

This article was published in the following Dove Press journal: International Journal of Nanomedicine

\author{
Yuping Zhao',* \\ Yanna Zhao (iD) ${ }^{1, *}$ \\ Qisan $M a^{\prime}$ \\ Bin Sun' \\ Qingpeng Wang' \\ Zhuang Ding $\mathbb{D}^{\prime}$ \\ Huaizhen Zhang ${ }^{2}$ \\ Xiuling $\mathrm{Chu}^{3}$ \\ Min Liu' \\ Zhengping Wang' \\ Jun $\operatorname{Han}\left(\mathbb{D}^{\prime}\right.$
}

'Institute of Biopharmaceutical Research, Liaocheng University, Liaocheng, Shandong 252059, People's Republic of China; ${ }^{2}$ School of Environment and Planning, Liaocheng University, Liaocheng, Shandong 252059, People's Republic of China; ${ }^{3}$ College of Agriculture, Liaocheng University, Liaocheng, Shandong 252059, People's Republic of China

*These authors contributed equally to this work
Correspondence: Yanna Zhao; Jun Han Institute of Biopharmaceutical Research, Liaocheng University, Liaocheng 252059, Shandong Province, People's Republic of China

Fax +86 6358239136

Email ynzhao201।@।63.com;

junhanmail@I63.com
Background: The combination of chemo-photodynamic therapy based on nano-technology has emerged as a preferable and promising measure for synergetic antitumor therapy.

Purpose: The aim of this study was expected to overcome most of the safety concerns from nano-carriers and improve the chemo-photodynamic synergistic antitumor efficacy.

Methods: Herein, we reported a facile and effective approach based on the self-assembly of chemotherapeutic agent 10-hydroxycamptothecin (HCPT) and photosensitizer chlorin e6 (Ce6) for preparing stably dual-functional nanorods (NRs).

Results: The chemical thermodynamic parameters obtained from isothermal titration calorimeter (ITC) and the microcosmic configuration snapshots acquired by molecular dynamics (MD) simulations verified that HCPT and Ce6 molecules tended to assemble with each other through various intermolecular forces. The as-prepared HCPT/Ce6 NRs possessed a relatively uniform size of around $165 \mathrm{~nm}$ and zeta potential of about $-29 \mathrm{mV}$, together with good stability in aqueous solution and freeze-dried state. In addition, both the extra- and intracellular reactive oxygen species (ROS) generation capacity of the NRs under laser irradiation was significantly enhanced compared with Ce6 injections. Moreover, the dual-functional HCPT/Ce6 NRs exhibited a substantial in vitro/in vivo synergistic antitumor efficacy under laser irradiation due to the integration of the two therapeutic modalities into one drug delivery system. Besides, no obvious hepatic or renal toxicity was observed in the NRs treatment groups.

Conclusion: Taken together, HCPT/Ce6 NRs demonstrated a powerful efficacy in chemophotodynamic therapy for breast cancer. Therefore, the carrier-free dual-functional NRs prepared in a facile and effective strategy might give inspiration for the development of combined antitumor therapy.

Keywords: carrier-free, self-assembly, chemo-photodynamic, dual-functional nanorods, combined antitumor therapy, synergistic antitumor efficacy

\section{Introduction}

According to the GLOBOCAN database, about 9.6 million cancer deaths have occurred in the year 2018, and the number of which will continue to rise and reach an estimated 13.1 million cancer deaths in $2030 .^{1,2}$ At present, the various therapies, such as surgery, radiotherapy, chemotherapy, and immunotherapy, have been widely used to improve the outcome and survival in patients with advanced cancer. ${ }^{3}$

Chemotherapeutic drug 10-hydroxycamptothecin (HCPT) has an extensive spectrum of anticancer activity by inhibiting the pathway of nuclear enzyme topoisomerase I and religation of the cleaved DNA strand. ${ }^{4}$ Over the last few decades, HCPT has been proven to be very effective in many malignancies, such as breast, colon, lung, hepatocellular, and 
ovarian cancers. $^{5-8}$ However, the lactonic ring of HCPT is unstable and can be reversibly hydrolyzed when exposed to alkaline solution, which significantly enhances its solubility but seriously reduces the therapeutic efficacy. ${ }^{9}$

In recent years, photodynamic therapy (PDT) has developed rapidly for the application in various cancer treatments due to its specific merits such as minimal invasiveness, reduced multi-drug resistance, and decreased systemic toxicity. ${ }^{10-12}$ The antitumor efficacy of PDT mainly relies on the photosensitizers (PSs), which could be activated by laser with appropriate wavelengths and generate singlet oxygen $\left({ }^{1} \mathrm{O}_{2}\right)$, one type of reactive oxygen species (ROS) arousing irreversible damage to the tumor cells. ${ }^{13} \mathrm{Ce} 6$, a promising second-generation PS with efficient ROS generation and strong absorption in the red spectral region (around $660 \mathrm{~nm}$ ) that allows deep tissue penetration, has been widely used in PDT. However, low selective target accumulation of $\mathrm{Ce} 6$ toward tumors and low bioavailability have indeed compromised cancer therapeutic efficacy and thus limited its clinical application. ${ }^{14}$

To date, the combinational therapy based on the nanosized drug delivery systems (NDDS) has been a prevalent and effective strategy, which can not only improve the solubility of hydrophobic drugs and enhance their targeting efficiency but also exhibit synergetic antitumor effect and minimal side effects compared with respective therapeutic method. ${ }^{15,16}$ Additionally, some biodegradable materials sensitive to the specific tumor microenvironment (such as acid, redox, and near-infrared responsiveness) are extensively researched in the field of NDDS area. For instance, Cao's group ${ }^{17,18}$ fabricated GNRs/SiO2/GO-PEG and Au/ $\mathrm{SiO} 2 / \mathrm{HAP}$ hybrid nanoparticles for multi-responsive drug delivery, all of which exhibited high drug loading efficiency, excellent $\mathrm{pH}$-/NIR-sensitivity as well as remarkable biocompatibility and biodegradability. Despite the remarkable improvement of multifunctional nano-medicine, some concerns are still worried about many other non-biodegradable inorganic polymers and carrier-induced toxicity during the process of degradation, metabolism, and excretion. ${ }^{19}$ Recently, carrier-free pure nanodrugs (PNDs) directly consisted of pharmaceutically active molecules, are expected to overcome most of the safety concerns from nano-carriers, as well as possess both nano-scale advantages and high drug payload simultaneously. ${ }^{20-22}$ Various PNDs composed of both chemotherapeutic drug and photosensitizer, such as co-assembled Ce6/DOX nano-spheres, UA-LA-ICG NPs, and ICG-EPI NPs, have been reported to exert enhanced synergetic antitumor effect. ${ }^{11,19,23}$ It is worth mentioning that Liang's group successfully fabricated a carrier-free
HCPT/Ce6 NRs by precipitation method, which exhibited significantly combined antitumor effect in vivo. ${ }^{15}$ However, the final nano-crystalline formulations contained organic solvent (DMSO) and sodium hydroxide, indicating a majority of HCPT probably existed as carboxylic salt form.

In this study, we rationally fabricated a dual-functional nano-drug delivery system by directly co-assembling HCPT with Ce6 via a combined reverse solvent precipitation method with high-pressure homogenization for synergetic chemo-photodynamic antitumor therapy (Scheme 1). The ultimately obtained HCPT/Ce6 NRs avoided the introduction of organic solvent and weak base, ensuring HCPT existing as lactone form. Besides, it was worth mentioning that the isothermal titration calorimeter (ITC) and molecular dynamics (MD) simulations were firstly introduced to analyze the molecular interactions between $\mathrm{Ce} 6$ and HCPT and reveal the co-assembly mechanism of the carrier-free nanorods (NRs). Furthermore, the physicochemical properties of the NRs, containing particle diameter, morphology, storage stability, release behavior, along with extra- and intracellular laser triggered ROS generation were evaluated in this work. Finally, both in vitro and in vivo tumor inhibition effects were analyzed. Conclusively, the HCPT/Ce6 NRs could be utilized as a potential nanodrug delivery system for synergetic chemo-photodynamic antitumor therapy.
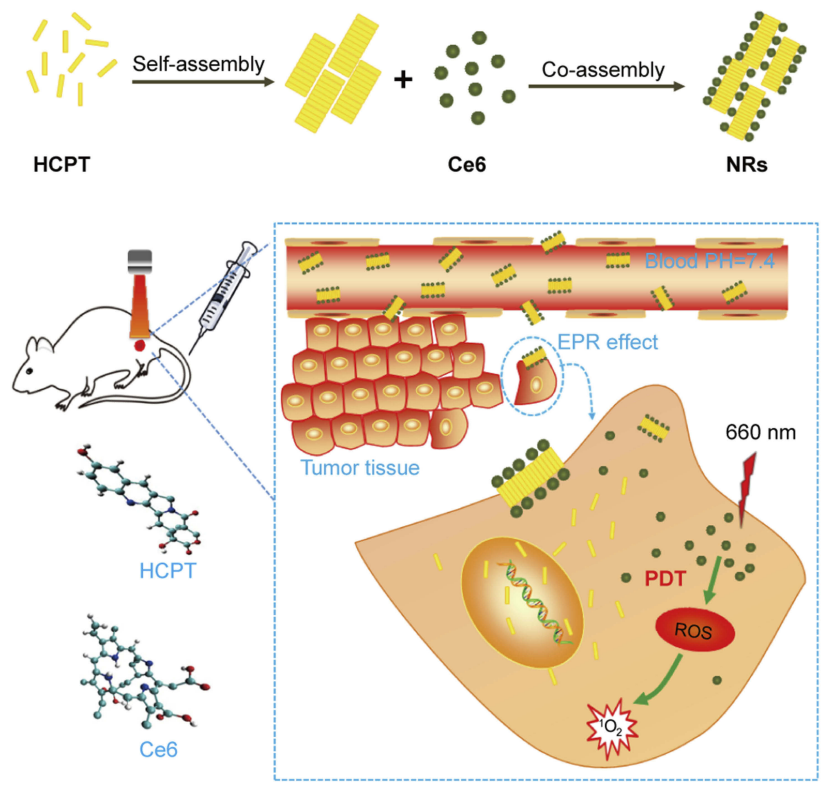

Scheme I Illustration for the preparation of carrier-free HCPT/Ce6 nanorods by co-assembling HCPT with $\mathrm{Ce} 6$ and the application of chemo-photodynamic synergetic antitumor therapy.

Abbreviations: HCPT, 10-hydroxycamptothecine; Ce6, Chlorin e6; NRs, nanorods; ROS, reactive oxygen species; PDT, photodynamic therapy; EPR, enhanced permeability and retention. 


\section{Materials And Methods \\ Materials}

HCPT and Ce6 were purchased from Meilunbio (Dalian, China). Dialysis bags (MW=8000-14,000) were bought from Shanghai Yuanye Bio-Technology Co., Ltd. (Shanghai, China). HPLC grade Acetonitrile (ACN, purity $\geq 99.9 \%$ ) was obtained from Adamas-beta Co., Ltd. (Shanghai, China), and Dimethyl sulfoxide (DMSO) came from Aladdin (Shanghai, China). The purified water was fabricated in laboratory using a Milli-Q A10 water generator (Millipore Co., Billerica, MA, USA). Other chemicals with analytical grade were purchased from a commercial company, which were used without further purification except as explained elsewhere.

4T1 cells (4T1 murine breast cancer cell line) were provided by Cell Culture Center, Institute of Basic Medical Sciences (Beijing, China). Roswell Park Memorial Institute-1640 (RPMI-1640) medium was manufactured by HyClone (Logan, UT, USA) and Fetal bovine serum (FBS) was supplied by Lonsa Science SRL (Montevideo, Uruguay). Penicillin and streptomycin solution $(100 \mathrm{U} / \mathrm{mL})$ were offered by Beyotime (Shanghai, China). The 3-(4,5-dimethylthiazol-2-yl)-2,5-diphenyltetrazolium bromide (MTT) was supplied by SigmaAldrich (St Louis, MO, USA). Hoechst (HOE) 33258 staining solution was purchased from Sangon Biotech (Shanghai, China), LysoTracker ${ }^{\circledR}$ green was purchased from KeyGen Biotech (Nanjing, China), DCFH-DA (2',7'-Dichlorodihydrofluorescein diacetate) was purchased from Meilunbio (Dalian, China) and Singlet Oxygen Sensor Green (SOSG) was purchased from Invitrogen by Thermo Fisher Scientific (Waltham, MA, USA).

Female BALB/c mice (SPF grade, 6 weeks) were afforded by Jinan Pengyue experimental animal breeding co. LTD (Jinan, China). All the mice were at controllable temperature $\left(25 \pm 2^{\circ} \mathrm{C}\right)$, relative humidity $(50-60 \%)$ and $12 \mathrm{hr}$ light-dark cycle with standard diet ad libitum for 1 week before experimentation. All the experimental procedures were carried out strictly in accordance with the Guidelines and Policies for Animal Experiments Ethical and Regulatory as approved by the Animal Ethics Committee of Liaocheng University.

\section{Isothermal Titration Calorimetry Measurement}

The thermodynamic parameters of molecular interaction between HCPT and Ce6 were detected by a Micro-Cal $\mathrm{ITC}_{200}$ isothermal titration calorimeter (GE Healthcare,
Madison, WI, USA). The reference cell of ITC was full of purified water, while the sample cell was added with Ce6 buffer solution $(0.4 \mathrm{mM}$ Ce6 in $50 \mathrm{mM}$ tris buffer containing $20 \%$ DMF). The titrant syringe was filled with HCPT buffer solution (2 mM HCPT in $50 \mathrm{mM}$ tris buffer containing $20 \%$ $\mathrm{DMF}$ ) and controlled by software. In each titration, 20 injections of $2 \mu \mathrm{L}$ HCPT buffer solution (note: the initial injection equal to $0.4 \mu \mathrm{L}$ ) were titrated into the sample cell at $150 \mathrm{~s}$ intervals; meanwhile, a stirring speed of $350 \mathrm{rpm}$ and system compensation power of $5 \mu \mathrm{cal} \cdot \mathrm{s}^{-1}$ were maintained throughout the experiment so as to ensure enough mixing after each injection. In order to ascertain the heat of dilution of HCPT and $\mathrm{Ce} 6$, control experiments were conducted by injecting isometric solvent into $\mathrm{Ce} 6$ buffer solution and HCPT buffer solution into solvent, respectively. The obtained correcting injection heat was plotted as a function of HCPT and Ce6 molar ratio and matched to various models in the Windowsbased Origin 7.0 software package supplied by the $\mathrm{ITC}_{200}$ calorimeter, which the peak of first injection was abnegated. From the experimental data by non-linear least-squares fitting, the combination number $(n)$, binding constants $(K)$, and standard molar enthalpy change $\left(\Delta H^{\circ}\right)$ were gained. According to the thermodynamic parameters $\left(K\right.$ and $\left.\Delta H^{\circ}\right)$, the $\Delta G^{\circ}$ (standard Gibbs free energy change), and $\Delta S^{\circ}$ (standard entropy change) could be calculated on the basis of the following equation: ${ }^{24}$

$$
\Delta G^{o}=-R T \ln K=\Delta H^{o}-T \Delta S^{o}
$$

\section{Molecular Dynamics Simulations Between HCPT And Ce6}

Molecular dynamics (MD) simulations were conducted to investigate how the HCPT and Ce6 molecules would interact with each other in aqueous solution. Firstly, the model was built by randomly placing $\mathrm{Ce} 6$ and HCPT molecules in an $8 \times 8 \times 8 \mathrm{~nm}^{3}$ box, and then the water was added into the box, achieving a density of about $1 \mathrm{mM}$. The MD simulations were performed using the GROMACS 4.6.3 package with the functions of the GROMOS53A6 force field, ${ }^{25,26}$ in which the united-atom description was adopted for all the molecules except water. The simple point charge/extend (SPC/E) model was used for water molecules. ${ }^{27}$ After minimizing the energies of the initial configurations with steepest descent method, a brief balance under NPT ensemble at $1 \mathrm{~atm}$ and $298 \mathrm{~K}$ using the V-rescale thermostat algorithm was performed to make the system volume stable. ${ }^{28}$ The Lennard-Jones interactions were applied for non-bonded potential truncation at a cut of $0.9 \mathrm{~nm}$ and the electrostatic interactions were calculated by the particle mesh Ewald method. ${ }^{29}$ Among the MD simulations, 
the trajectories were stored every 2 fs and trajectories (molecular interactions and binding sites) were visualized using VMD software.

\section{Preparation Of HCPT/Ce6 NRs}

The HCPT/Ce6 NRs were prepared by combining reverse solvent precipitation method with high-pressure homogenization technique. Primarily, HCPT (7.5 mg) and Ce6 (4.1 $\mathrm{mg}$ ) were dissolved into $0.5 \mathrm{~mL}$ DMF for preparing stock solution, respectively. The HCPT organic solution was added dropwise into $15 \mathrm{~mL}$ deionized water with continuous ultrasonic $(100 \mathrm{~W})$ in an ice bath. Afterwards, the Ce6 liquor was injected into above solution at the same conditions, followed by ultrasound for 5 mins after injection completed. Then, the mixture was transferred into a preprocessed dialysis bag $(\mathrm{MW}=8000-14,000)$ immersed into deionized water $(4 \times 1 \mathrm{~L})$ and dialyzed for $2 \mathrm{hrs}$ with continuous stirring to remove DMF and free drugs, and then homogenized (1000 bar) for 4.5 mins using a PhD D-3L homogenizer (PhD Technology LLC, Bloomington, MN, USA). The same method was used to prepare other formulations with different HCPT/Ce6 molar ratios.

The drug loading content (DLC) of HCPT/Ce6 NRs was determined and calculated according to the following formula:

$$
D L C(\%)=\frac{\text { weight of drug in nanorods }}{\text { total weight of nanorods }} \times 100 \%
$$

\section{Characterization Of HCPT/Ce6 NRs}

\section{The Size And Zeta-Potential Measurement}

The particle diameter, size distribution, zeta-potential, and poly-dispersity index (PDI) values of $\mathrm{HCPT} / \mathrm{Ce} 6$ NRs were tested by dynamic light scattering (DLS) with a Malvern Nano ZSP (Malvern Instruments, Malvern, UK) with standard laser $(10 \mathrm{mV}, \lambda=633 \mathrm{~nm})$ at $25^{\circ} \mathrm{C}$.

\section{Transmission Electron Microscope (TEM) And Scanning Electron Microscope (SEM)}

The morphology feature of HCPT/Ce6 NRs was performed using a JEM-2100 TEM (JEOL Ltd., Tokyo, Japan) with an accelerating voltage $(80 \mathrm{kV})$. A drop of HCPT/Ce6 NRs was placed on copper grid and dried in the air. Then, the NRs were dyed with uranyl acetate solution $(1 \%, \mathrm{w} / \mathrm{v})$ for $2 \mathrm{~min}$ and analyzed by TEM.

To further evaluate the morphology of HCPT/Ce6 NRs, HCPT powder and Ce6 powder, the S-4800 SEM (Hitachi Limited., Tokyo, Japan) was applied. Samples were spread on a metal stub with double-sided adhesive carbon tap, then sputter-coated with a conductive thin layer of gold-palladium $(\mathrm{Au} / \mathrm{Pd})$ alloy in an argon atmosphere for $1 \mathrm{~min}$. An accelerating voltage of $5 \mathrm{kV}$ was used for the observation and analysis.

\section{Ultraviolet Visible (UV-Vis) And Fluorescence (FL) Spectra}

UV absorption spectra of HCPT/Ce6 NRs solution were detected using a Scinco S-3100 UV-vis spectrometer (Scinco, Seoul, Korea) at room temperature. HCPT injection and $\mathrm{Ce} 6$ injection were also implemented as the comparison under the same circumstances.

Fluorescence (FL) spectra were examined by a Hitachi F-7000 fluorescence spectrophotometry (Hitachi, Ltd., Tokyo, Japan) with the excitation at $346 \mathrm{~nm}$ (for HCPT) and $405 \mathrm{~nm}$ (for Ce6). The samples were HCPT injection, Ce6 injection, and HCPT/Ce6 NRs, all of which were diluted to certain concentration (equivalent to $1.8 \mu \mathrm{g} / \mathrm{mL}$ HCPT and $1 \mu \mathrm{g} / \mathrm{mL} \mathrm{Ce}$ ) by PBS ( $\mathrm{pH}=7.4)$ before testing.

\section{Fourier-Transform Infrared Spectroscopy (FT-IR)}

Infrared (IR) spectrum was collected by a Nicolet 6700 FTIR spectrometer (Thermo Scientific, Waltham, MA, USA) using Potassium Bromide pellets technique in the region $500-4000 \mathrm{~cm}^{-1}$. The samples were HCPT powder, Ce6 powder, HCPT/Ce6 physical mixture, and HCPT/Ce6 NRs.

\section{Powder X-Ray Diffraction (XRD)}

The XRD patterns of HCPT powder, Ce6 powder, HCPT/ Ce6 physical mixture, and HCPT/Ce6 NRs were executed by a D8 Advance X-ray powder diffractometer (Bruker Inc., Rheinstetten, Germany) with Ni-filtered $\mathrm{Cu} \mathrm{K \alpha}(1.54$ $\AA$ ) at radiation $40 \mathrm{kV}$. The powders were tested over a $2 \theta$ range from $5^{\circ}$ to $50^{\circ}$ with electric current of $200 \mathrm{~mA}$.

\section{Stability Study}

The HCPT/Ce6 NRs were stored at $4{ }^{\circ} \mathrm{C}$ for 21 days in order to study the storage stability. The particle size and zetapotential of the NRs were detected at $0,1,3,5,7,14$, and 21 days, and the experiments were performed in triplicates.

The stability study of lyophilized samples was also investigated using a BTP 31 freeze dryer (SP Scientific, Warminster, PA, USA) in ultrahigh vacuum degrees (195200 ubar) with the condenser temperature $\left(-50\right.$ to $\left.-55^{\circ} \mathrm{C}\right)$. The lyophilized NRs were prepared by adding different cryoprotectants (sucrose, lactose, mannitol, poloxamer, glycine, trehalose, poloxamer, and polyvinyl pyrrolidone) in varying proportions $(0.5,1,2,4,6,8$, and $10 \%)$. After lyophilization, $\mathrm{HCPT} / \mathrm{Ce} 6 \mathrm{NRs}$ were stored at room 
temperature and re-dissolved in $1 \mathrm{~mL}$ water under slight ultrasonication and characterized by DLS at predetermine days. All the experiments were performed in triplicates and expressed with the mean plus standard deviation $( \pm \mathrm{SD})$.

\section{In Vitro Drug Release Behavior}

The drug release behavior of HCPT/Ce6 NRs was studied using a BSD TX270 shaker (Boxun, Shanghai, China). Concisely, $2 \mathrm{~mL}$ of HCPT/Ce6 NRs solution with the HCPT concentration of $100 \mu \mathrm{g} / \mathrm{mL}$ and $\mathrm{Ce} 6$ concentration of $55.5 \mu \mathrm{g} /$ $\mathrm{mL}$ was imported into a dialysis bag $(\mathrm{MW}=8000-14,000)$, which was then immersed into $100 \mathrm{~mL}$ PBS $(\mathrm{pH}=7.4)$ containing $0.5 \% \mathrm{w} / \mathrm{v}$ sodium dodecyl sulfate (SDS) at $37^{\circ} \mathrm{C}$ with the shaking speed of $100 \mathrm{rpm} / \mathrm{min}$. HCPT injection and Ce6 injection were also implemented as the comparison under the same circumstances. A total of $4 \mathrm{~mL}$ of menstrua was withdrawn at different time points for analysis, and the same volume of fresh media was supplemented. The concentration of released drug was measured by a Dionex Ultimate 3000 HPLC (DIONEX, Sunnyvale, CA, USA) with Variable Wavelength Detector (VWD) for HCPT and a Hitachi F-7000 fluorescence spectrophotometry for Ce6. Standard curves were built-up, respectively, for HCPT and Ce6, and all the correlation coefficients were more than 0.999 (Table S1A). All the release experiments were conducted in triplicates.

\section{In Vitro Cytotoxic Effect}

The in vitro cytotoxicity was investigated by MTT assay against 4T1 cells. Briefly, cells in log phase were seeded into 96-well plates $\left(1 \times 10^{4}\right.$, per well), and incubated in RPMI-1640 medium containing 10\% fetal bovine serum (FBS) and $1 \%$ penicillin-streptomycin solution with $5 \% \mathrm{CO}_{2}$ at $37^{\circ} \mathrm{C}$ for 24 hrs. Then, the 4T1 cells were cultured with HCPT injection, Ce6 injection, and HCPT/Ce6 NRs of predetermined concentrations for $4 \mathrm{hrs}$. Afterwards, the plate was irradiated by laser ( $5 \mathrm{~mW}, 2$ mins each well) using a $660 \mathrm{~nm}$ laser source for PDT treatment groups. After further incubation for $48 \mathrm{hrs}, 20 \mu \mathrm{L}$ of MTT solution ( $5 \mathrm{mg} / \mathrm{mL}$ in PBS) was added per well with incubating for another $4 \mathrm{hrs}$. Then, the supernatant was removed and DMSO $(150 \mu \mathrm{L})$ was added to each well, followed by shaking the plates a few times. The optical density (OD) of each well was tested by a synergy $\mathrm{H} 1$ multifunctional microporous plate detector (BioTek instruments, Winooski, VT, USA) at the wavelength of $570 \mathrm{~nm}$. The viability of cells was calculated using the following equation, while the half-maximal inhibitory concentration $\left(\mathrm{IC}_{50}\right)$ was then simulated:

$$
\text { Cell viability }(\%)=O D_{\text {Sample }} / O D_{\text {Control }} \times 100 \%
$$

The combination index (CI) was also analyzed to evaluate the synergy of HCPT and Ce6 inside NRs according to the formula previously reported. ${ }^{30,31}$

$$
C I=\left[C_{A, x} / I C_{x, A}\right]+\left[C_{B, x} / I C_{x, B}\right]
$$

When the drug effect $\mathrm{x} \%$ was obtained in combination treatment, $\mathrm{C}_{\mathrm{A}, \mathrm{x}}$ and $\mathrm{C}_{\mathrm{B}, \mathrm{x}}$ represent the concentrations of drugs $\mathrm{A}$ and $\mathrm{B}$, respectively. When the same drug effect was acquired in monotherapy treatment, $\mathrm{IC}_{\mathrm{x}, \mathrm{A}}$ and $\mathrm{IC}_{\mathrm{x}, \mathrm{B}}$ represent the concentrations of drugs $\mathrm{A}$ and $\mathrm{B}$, respectively. The assessment method of combination index was additive effects $(\mathrm{CI}=1)$, synergism $(\mathrm{CI}<1)$ and antagonism $(\mathrm{CI}>1)$.

\section{In Vitro Cell Uptake Experiment}

The cellular uptake experiments of HCPT/Ce6 NRs were characterized in 4T1 cells using fluorescence microscope imaging system and high-performance liquid chromatography with fluorescence detection (HPLC-FLD). For the observation by fluorescence microscope, 4T1 cells $\left(2 \times 10^{4}\right.$, per well) were seeded into 24-well plates and cultured for 24 hrs at a cell incubator. The cells were first incubated with NRs (equivalent to $18 \mu \mathrm{g} / \mathrm{mL}$ HCPT and $10 \mu \mathrm{g} / \mathrm{mL}$ Ce6) for 4 hrs, and then rinsed by PBS and counterstained with Hoechst 33258 (10 mM) for 10 mins. After washing thrice with PBS, LysoTracker ${ }^{\circledR}$ Green $(1 \mu \mathrm{M})$ was added to per well. Followed by incubation at $37^{\circ} \mathrm{C}$ for $30 \mathrm{mins}$, the cells were bathed for 3 times, fixed with $4 \%$ paraformaldehyde solution for $15 \mathrm{mins}$ at room temperature, and visualized by a Nikon Eclipse Ti-s inverted fluorescence microscope (Nikon Ltd, Tokyo, Japan). The NRs were emerged by the red signals from $\mathrm{Ce} 6$, while cytoplasm was presented by green signals from LysoTracker $^{\circledR}$ Green and nuclei was checked in blue from Hoechst 33258.

For the quantitative determination by HPLC-FLD, 4T1 cells $\left(5 \times 10^{4}\right.$, per well) were seeded into 24 -well plates and cultured at $37^{\circ} \mathrm{C}$ for $48 \mathrm{hrs}$. The NRs at predetermined concentration were joined into 24-well plates. After the plates were incubated at definite time, the supernatant was removed, and the cells were washed three times with PBS. Then, the cells were digested by trypsin, collected after centrifugation, and suspended with $0.2 \mathrm{~mL}$ cell lysis buffer to extract the intracellular drugs. The above suspension was mixed with $0.2 \mathrm{~mL} \mathrm{ACN}$, followed by centrifuging at $10,000 \mathrm{rpm}$ for $30 \mathrm{mins}$ to collect the supernatant liquor. 
The supernatant was filtered using filter $(0.22 \mu \mathrm{m})$ and injected $(50 \mu \mathrm{L})$ into an HPLC system (UltiMate 3000, Dionex) with a fluorescence detector (Excitation/emission=346 nm/536 nm) and an Agilent C18 column $(4.6 \mathrm{~mm} \times 250 \mathrm{~mm}, 5 \mathrm{~mm})$ for analysis. Acetonitrile $(\mathrm{ACN}) /$ water with $0.1 \%$ acetic acid $(28 / 72, \mathrm{v} / \mathrm{v})$ was employed as a mobile phase, and the flow rate was 0.8 $\mathrm{mL} / \mathrm{min}$. All the experiments were carried out in triplicate. A standard curve was built-up and the correlation coefficient was more than 0.999 (Table S1B).

\section{Determination Of Singlet Oxygen}

Singlet oxygen sensor green reagent (SOSG) was used to determine the singlet oxygen $\left({ }^{1} \mathrm{O}_{2}\right)$ produced by photosensitizer Ce6. A total of $3 \mu \mathrm{L}$ SOSG solution was added into PBS, Ce6 injection, HCPT injection and HCPT/Ce6 NRs (equivalent to $18 \mu \mathrm{g} / \mathrm{mL} \mathrm{HCPT}$ and $10 \mu \mathrm{g} / \mathrm{mL} \mathrm{Ce}$ ) with the final concentration of SOSG $1 \mu \mathrm{M}$, respectively. After the four mixtures were irradiated severally at different time periods with a laser $(660 \mathrm{~nm}, 5 \mathrm{~mW})$, the fluorescence intensity of internal oxidation product SOSG endoperoxides (SOSG-EP) was measured using a fluorescence spectrophotometry (F-7000, Hitachi) with the excitation at $498 \mathrm{~nm}$ and emission at $525.8 \mathrm{~nm}$.

For intracellular reactive oxygen species (ROS) generation detecting, 4T1 cells $\left(1 \times 10^{4}\right.$ per well $)$ were seeded into 96-well plates with volume of $100 \mu \mathrm{L}$ each well. After $24 \mathrm{hrs}$ of incubation $\left(37^{\circ} \mathrm{C}\right.$ and $\left.5 \% \mathrm{CO}_{2}\right)$, the supernatant was discarded, and then cells were further incubated with medium containing HCPT injection, Ce6 injection and HCPT/Ce6 NRs at various Ce6 concentrations. After incubation for 4 hrs, the cells were washed with serum-free media and cultured with $10 \mu \mathrm{M}$ DCFH-DA in RPMI 1640 for 30 mins, followed by exposure to a $660 \mathrm{~nm}$ laser $(5 \mathrm{~mW})$ for certain time. Then, ROS production was monitored with the microplate reader (synergy $\mathrm{H} 1$, BioTek) $\left(\mathrm{E}_{\mathrm{x}}=480 \mathrm{~nm}\right.$ and $\mathrm{E}_{\mathrm{m}}=530$ $\mathrm{nm}$ ), followed by fluorescence microscope imaging with an inverted microscope (Eclipse Ti-s, Nikon). All the images were marked at the same condition.

\section{In Vivo Therapeutic Efficacy}

The in vivo tumor suppression effect of HCPT/Ce6 NRs was assessed with the $4 \mathrm{~T} 1$-tumor-bearing BALB/c mice models. Briefly, 5-6 weeks old female mice (18-22 g) were subcutaneously injected for $0.2 \mathrm{~mL}$ cell suspension (around $5 \times 10^{6} 4 \mathrm{~T} 1$ cells) in the armpit. When tumors were greater than $50 \mathrm{~mm}^{3}$ (5 days after implantation), the mice were divided into six groups ( $\mathrm{n}=6$ per group) at random: (1) normal saline (NS) as a control, (2) HCPT injection (5.0 mg/kg), (3) Ce6 injection (Ce6 $2.77 \mathrm{mg} / \mathrm{kg}$ ) with irradiation, (4) HCPT+Ce6 injection (equal to $5.0 \mathrm{mg} / \mathrm{kg}$ and Ce6 $2.77 \mathrm{mg} / \mathrm{kg}$ ) with irradiation, (5) HCPT/Ce6 NRs (equal to $5.0 \mathrm{mg} / \mathrm{kg}$ and Ce6 $2.77 \mathrm{mg} / \mathrm{kg}$ ) without irradiation, and (6) HCPT/Ce6 NRs with irradiation. The treatment lasted for 10 days through tail vein injection with $0.2 \mathrm{~mL}$ different formulations (every 2 days for 5 times). For PDT therapy groups, $4 \mathrm{hrs}$ after injection, the mice were irradiated by a $660 \mathrm{~nm}$ laser $(5 \mathrm{~mW})$ for 30 mins. The tumor size (lengths and widths) and body weight were measured daily using digital caliper and electronic balance, respectively. The tumor volume was calculated on the basis of the following formula: $\mathrm{V}\left(\mathrm{mm}^{3}\right)=$ length $\times$ width $^{2} / 2$, and tumor inhibition rate (TIR) was also expression according to the formula: TIR $(\%)=$ (1-tumor weight of treated group/tumor weight of control group $) \times 100 \%$. After the experiment, the plasma was separated from the blood sample by centrifugation and then stored at $-80^{\circ} \mathrm{C}$ for studying the activity of aspartate aminotransferase (AST) and alanine aminotransferase (ALT) as well as the level of blood urea nitrogen (BUN) and creatinine (CRE).

\section{In Vivo Study Of Toxicity}

For evaluating the toxicity of NRs and injections of HCPT and $\mathrm{Ce} 6$, the tumors and main organs (heart, liver, spleen, lung, and kidney) were also collected, washed using $0.9 \%$ saline solution, dried with filter paper, and accurately weighed. The intact organs were fixed with $4 \%$ paraformaldehyde for hematoxylin-eosin staining (H\&E stain) analysis.

\section{Statistical Analysis}

Statistical analyses were done by SPSS Statistics 17.0 software. Independent-samples Student's $t$-test was used to evaluate the differences between groups, and $\mathrm{P}<0.05$ was statistically significant. The results were expressed as the mean value \pm standard deviation (SD).

\section{Results And Discussion Isothermal Titration Calorimetry Measurement}

The molecular interaction between HCPT and $\mathrm{Ce} 6$ molecules was preliminary investigated by ITC technique, which could directly measure the thermodynamic characteristics (the changes of enthalpy, entropy, and standard free energy) of interactional processes in solution. ${ }^{32}$ Figure 1 showed the isothermal titrations calorimetric curves of HCPT into $\mathrm{Ce} 6$ in buffer solution (50 $\mathrm{mM}$ tris containing $20 \% \mathrm{DMF}$ ) at $298.15 \mathrm{~K}$ and two Set of Sites model apparently fitted as 


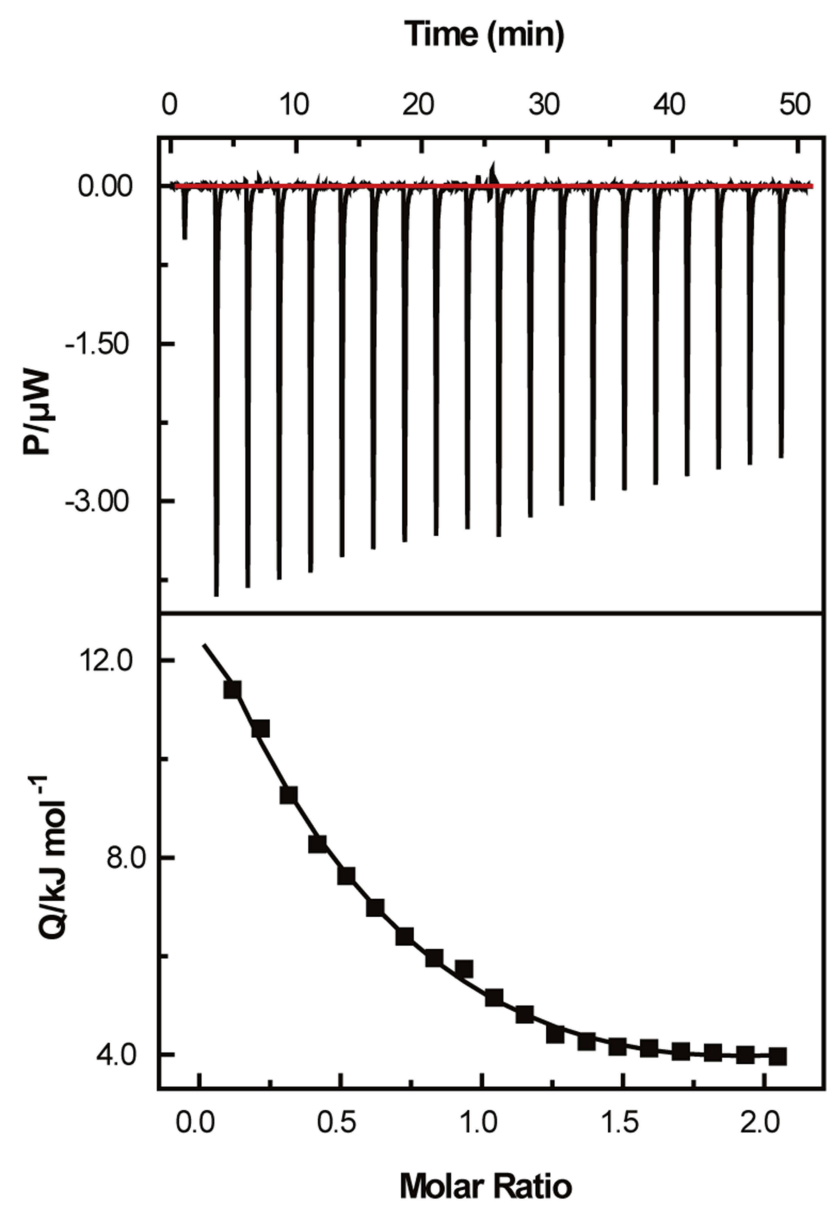

Figure I Isothermal titration calorimetry profiles for the binding of HCPT with Ce6 in buffer ( $50 \mathrm{mM}$ Tris containing $20 \% \mathrm{DMF}$ ) at $298.15 \mathrm{~K}$. The upper panel represents the raw data for the titration of HCPT into Ce6 solution. The bottom panel represents the integrated heat data obtained from the raw data after deducting the heat of dilution, in which the solid square and solid line panel indicate the experimental data and the best curve fitting to the experimental data, respectively.

Abbreviations: HCPT, 10-hydroxycamptothecine; Ce6, Chlorin e6.

the smooth solid line passed through all the experimental points. To further study the mechanism of the affinity between HCPT and Ce6, thermodynamic parameters $\left(\Delta H^{\circ}\right.$, $\Delta S^{\circ}$, and $\Delta G^{\circ}$ ) were obtained from the ITC data and summarized in Table 1 . The values of $\Delta G^{\circ}$ were negative throughout the titration process, demonstrating that the conjugation between $\mathrm{HCPT}$ and $\mathrm{Ce} 6$ was spontaneous. ${ }^{33}$ Moreover, all the entropy changes were positive $\left(\Delta S_{\mathrm{i}}>0\right)$, as well, the contribution of $-T \Delta S^{\circ}$ to $\Delta G^{\circ}$ was larger than that of $\Delta H^{\circ}$, indicating the binding of HCPT to $\mathrm{Ce} 6$ was primarily entropically driven. Hydrophobic interactions, hydrogen bonds, electrostatic attraction, and van der Waals force were the main driving forces between drug molecules. ${ }^{34}$ Furthermore, combining the enthalpy with entropy changes, interactions between HCPT and Ce6 molecules were mainly hydrophobic interaction $\left(\Delta H_{1}>0, \Delta S_{1}>0 ; \Delta H_{3}>0, \Delta S_{3}>0\right)$ and electrostatic attraction $\left(\Delta H_{2}<0, \Delta S_{2}>0\right)$. So it was confirmed that the assembly mechanism of the NRs was mainly the hydrophobic interaction (the $\pi-\pi$ conjugation of the ring structure) and the electrostatic interaction between HCPT and Ce6 molecules. ${ }^{35,36}$ The association constant $(n=3)$ indicated that one molecule of $\mathrm{Ce} 6$ probably combined with three HCPT molecules. Therefore, when HCPT/Ce6 NRs were prepared, the molar ratios of HCPT and $\mathrm{Ce} 6$ would be set as $1: 1,2: 1,3: 1$, and $4: 1$.

\section{Molecular Dynamics (MD) Simulations Between HCPT And Ce6}

The interaction behavior of HCPT and Ce6 in aqueous solution was further verified by the MD simulations. MD simulations configuration exhibited that, when the two types of molecules initially arranged apart in water (Figure 2A), HCPT was first adsorbed and rapidly self-assembled with each other within 10 ns (Figure 2B), and then the Ce6 molecule was co-assembled with HCPT molecules at a ratio of 2:1 (HCPT/Ce6) after $40 \mathrm{~ns}$ (Figure 2C). The result slightly differed from the ITC outcome, which might be due to the MD simulations were dynamic simulating process. As HCPT tended to self-assemble with each other initially and then coassemble with Ce6, the preparation of the dual-drug NRs was modified by adding HCPT primarily, followed by adding Ce6

Table I The Thermodynamic Parameters And Combination Number For The Binding Of HCPT With Ce6 Obtained From The Analysis Of Isothermal Titration Calorimetry At 298.15 K

\begin{tabular}{|c|c|c|c|c|c|}
\hline $\mathrm{n}^{\mathrm{a}}$ & Number & $\begin{array}{l}K^{b} \\
\left(\times 10^{4} M^{-1}\right)\end{array}$ & $\begin{array}{l}\Delta H^{\mathrm{o}, \mathrm{c}} \\
\left(\mathrm{kJ} \cdot \mathrm{mol}^{-1}\right)\end{array}$ & $\begin{array}{l}T \Delta S^{o, d} \\
\left(k J \cdot \mathrm{mol}^{-1}\right)\end{array}$ & $\begin{array}{l}\Delta G^{\circ, e} \\
\left(\mathrm{~kJ} \cdot \mathrm{mol}^{-1}\right)\end{array}$ \\
\hline \multirow[t]{3}{*}{3} & I & $19.40 \pm 3.7$ & $12.75 \pm 0.23$ & $42.93 \pm 0.52$ & $-30.18 \pm 0.47$ \\
\hline & 2 & $8.34 \pm 2.30$ & $-4.29 \pm 0.43$ & $23.80 \pm 0.81$ & $-28.09 \pm 0.68$ \\
\hline & 3 & $3.98 \pm 0.92$ & $11.40 \pm 0.66$ & $37.65 \pm 0.87$ & $-26.25 \pm 0.57$ \\
\hline
\end{tabular}

Notes: ${ }^{a}$ Combination number represented that the molar ratio of HCPT to Ce6 was 3:I. ${ }^{\mathrm{b}}$ Binding constants. ${ }^{\mathrm{C}}$ Standard enthalpy changes. ${ }^{\mathrm{d}}$ Standard entropy changes. e Standard free energy changes.

Abbreviations: HCPT, 10-hydroxycamptothecine; Ce6, Chlorin e6. 


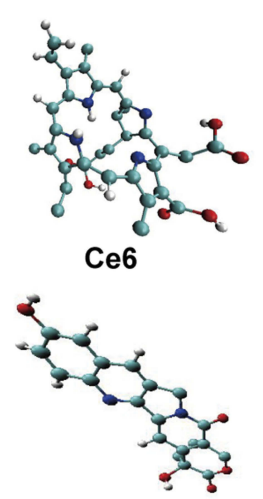

HCPT

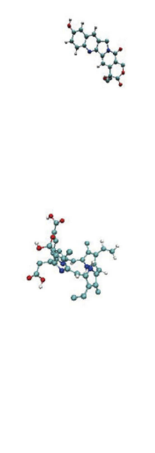

A

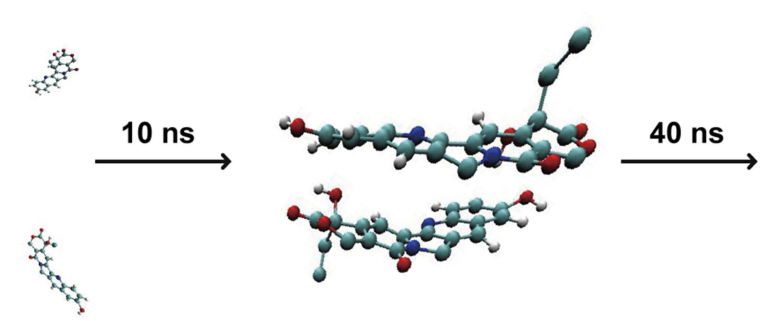

B

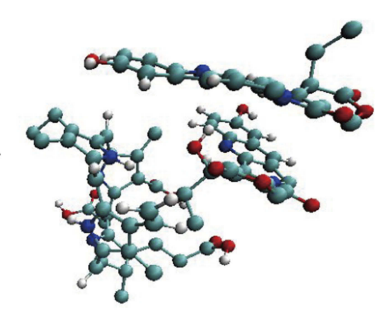

C

Figure 2 Molecular dynamics simulations for HCPT and Ce6 molecules.

Notes: (A) Two types of molecules initially arranged apart in water. (B) The self-assembly of HCPT molecules in water after $10 \mathrm{~ns}$. (C) The co-assembly of HCPT with Ce6 molecules in water after 40 ns. The software was VMD.

Abbreviations: HCPT, 10-hydroxycamptothecine; Ce6, Chlorin e6; ns, nanosecond.

as stabilizer to suppress crystallization and form stable complex. $^{37}$

\section{Preparation Of HCPT/Ce6 NRs}

In this study, different molar ratios $(\mathrm{HCPT} / \mathrm{Ce} 6=1: 1,2: 1$, $3: 1$ and $4: 1$ ) between two pure drugs were applied to obtain the optimal formulation of NRs. Table 2 showed that the NRs with the molar ratio of $3: 1$ exhibited the smallest particle size of $165.9 \pm 2.1 \mathrm{~nm}$ (Figure $3 \mathrm{~A}$ ) with a relatively narrow size distribution $(\mathrm{PDI}=0.179 \pm 0.026)$ compared with other ratios. Moreover, the storage stability results (Table S2) of molar ratios (HCPT/Ce6=1:1, 2:1, 3:1 and $4: 1)$ at $4^{\circ} \mathrm{C}$ for 14 days showed that there was no significant difference between the molar ratio $3: 1$ and $4: 1$, while the relatively small particle size and narrow size distribution were observed compared with other ratios (HCPT/Ce6=2:1 and 1:1). Hence, combined with the results of ITC and storage stability, the NRs with the molar ratio $3: 1$ of HCPT to $\mathrm{Ce} 6$ were selected as the optimum formula for subsequent investigations. According to this optimum formulation, the DLC (\%) of two components in NRs was

Table 2 Results Of HCPT/Ce6 NRs Prepared Via Different Drug/Molar Ratio Of HCPT To Ce6

\begin{tabular}{|l|l|l|l|}
\hline Drug/Molar Ratio & $\mathbf{D}_{\boldsymbol{h}}(\mathbf{n m})$ & PDI & ZP $(\mathbf{m V})$ \\
\hline $4: 1$ & $200.0 \pm 4.3$ & $0.194 \pm 0.024$ & $-30.9 \pm 1.4$ \\
$3: 1$ & $165.9 \pm 2.1$ & $0.179 \pm 0.026$ & $-28.9 \pm 1.1$ \\
$2: 1$ & $170.9 \pm 1.8$ & $0.205 \pm 0.010$ & $-23.8 \pm 0.4$ \\
$\mathrm{I}: 1$ & $174.7 \pm 2.6$ & $0.211 \pm 0.017$ & $-23.8 \pm 1.4$ \\
\hline
\end{tabular}

Notes: All results are presented as mean $\pm S D(n=3)$. $D_{h}$, mean hydrodynamic diameter. Abbreviations: HCPT, 10-hydroxycamptothecine; Ce6, Chlorin e6; NRs, nanorods; ZP, zeta potential; PDI, poly-dispersity index. determined as HCPT 75.18\% and Ce6 24.12\% (the molar ratio of HCPT to $\mathrm{Ce} 6$ was around $3: 1$ ).

Referring to previous reports, the suitable particle size of less than $200 \mathrm{~nm}$ was beneficial for the nanoparticles to avoid the reticuloendothelial system (RES) uptake and achieve passive tumor targeting based on the enhanced permeability and retention (EPR) effect. ${ }^{38}$ Besides, the zeta potential of HCPT/Ce6 NRs (HCPT/ Ce6=3:1) was $-28.9 \pm 1.1 \mathrm{mV}$ (Figure 3B), which indicated the NRs were apt to show negligible hemolytic and cytotoxic properties, display lower non-specific protein adsorption and exhibit a slow or reduced opsonization by RES. ${ }^{39}$

\section{Characterization Of HCPT/Ce6 NRs} TEM And SEM

The morphology of HCPT/Ce6 NRs was observed using TEM, where it could be seen that the dual-drug NRs were mainly regular rod-like nanostructures (Figure 4A). The average size of HCPT/Ce6 NRs was approximately $348 \pm 27 \mathrm{~nm}$ in length and $88 \pm 12 \mathrm{~nm}$ in diameter based on the TEM images. The difference between particle size obtained by TEM and dynamic light scattering technique (DLS) should be due to that DLS generally analyzed the equivalent spherical particle size rather than the real size of the NRs. According to the previous reports, ${ }^{40,41}$ in general, rod-like nanostructures reveal very good pharmacokinetic characteristics and efficiency in drug delivery owing to their multiple endocytic mechanisms, high internalization rates, valid adhesion to the cell surface, and more rapid penetration to the tumors. Moreover, the typical rod-like nanostructures could enhance their stability in aqueous system due to large specific surface area. ${ }^{42}$ Most worthy of 
A

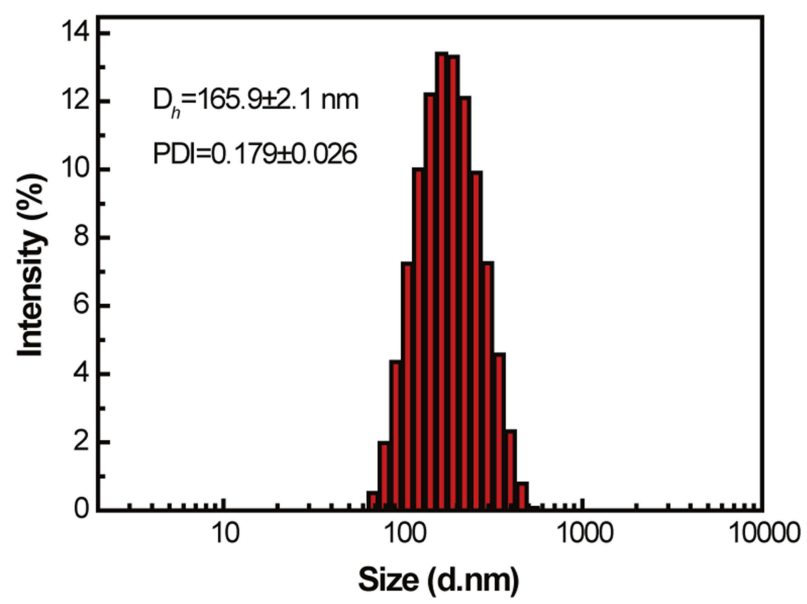

C

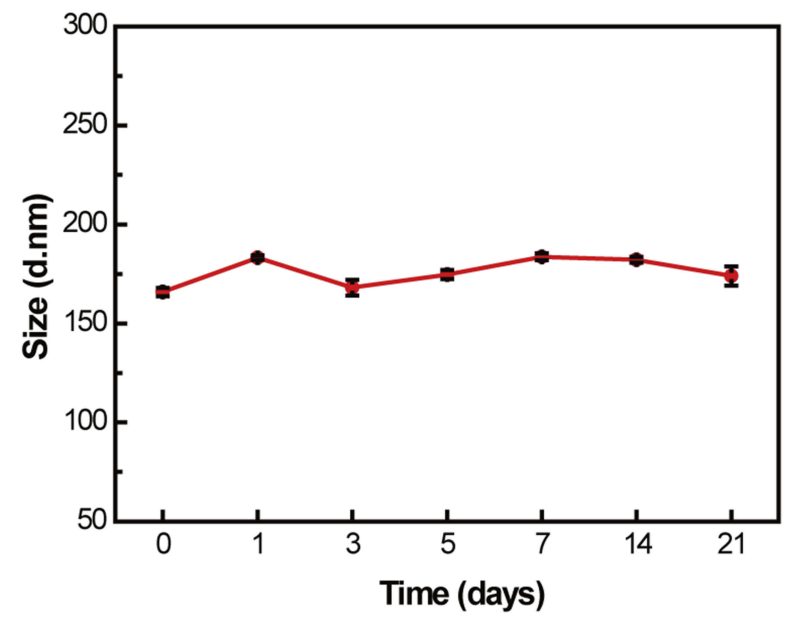

B

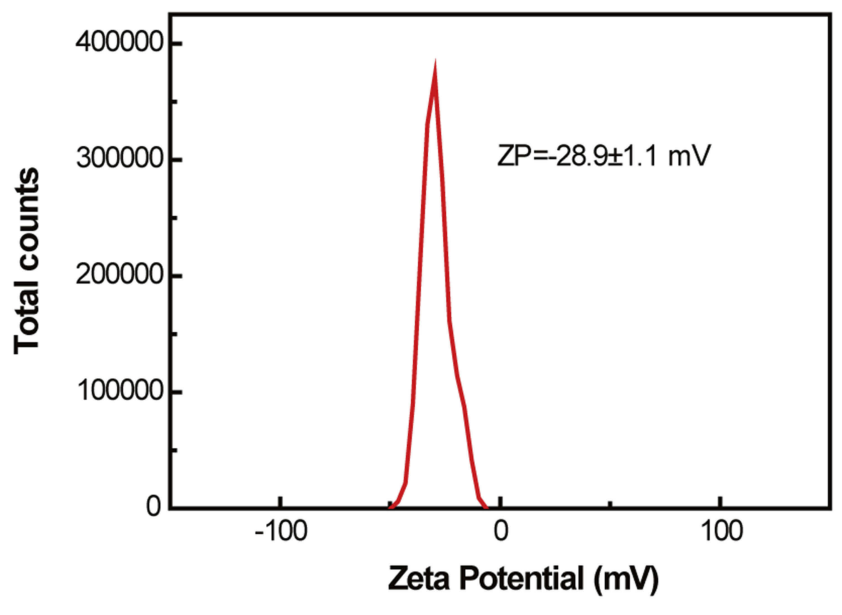

D

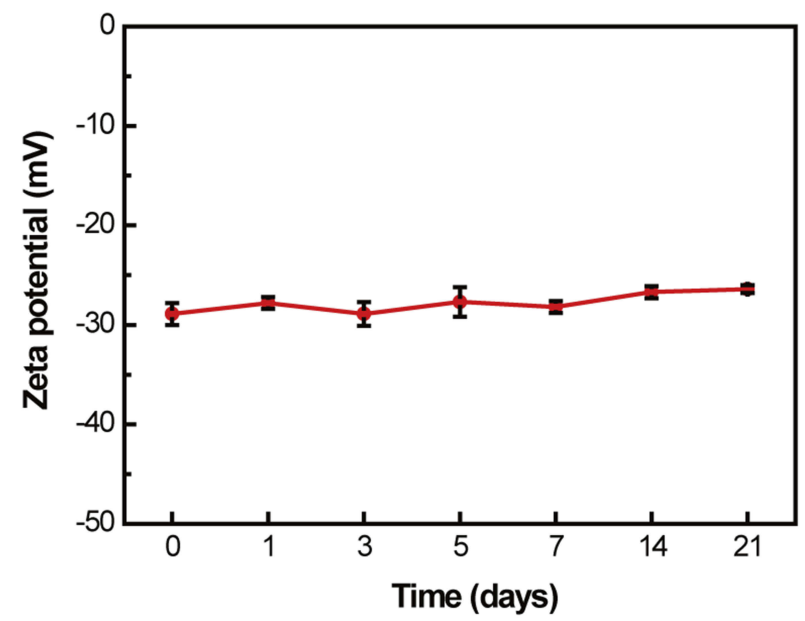

Figure 3 Characterization of dual-functional HCPT/Ce6 nanorods.

Notes: Distribution of $(\mathbf{A})$ particle size and $(\mathbf{B})$ zeta potential; stability of $(\mathbf{C})$ particle size and $(\mathbf{D})$ zeta potential as a function of time $(0$ to 21 days $)$ in purified water. $D_{h}$, mean hydrodynamic diameter.

Abbreviations: ZP, zeta potential; HCPT, I0-hydroxycamptothecine; Ce6, Chlorin e6; PDI, poly-dispersity index.

mention was that, for our functional photodynamic agents, the large surface area of the rod-like outer surface could also raise the amount of light absorption, which would make for a higher absorption coefficient and enhanced photodynamic therapy. $^{43,44}$

SEM was further utilized to compare the morphology of HCPT/Ce6 NRs with HCPT and Ce6 bulk powder. The SEM micrographs (Figure 4B) showed that the NRs in freeze-dried state were still mainly regular rod-like nanostructures, and the results were consistent with that of TEM. However, HCPT bulk powder presented mostly of agminated nubbly drug crystals with larger size $(>1$ $\mu \mathrm{m}$, Figure 4C), and Ce6 bulk powder exhibited irregular shapes, composed mostly of unformed drug crystals (Figure 4D). These results suggested that the HCPT and
Ce6 molecules indeed co-assembled into regular NRs via molecular interactions between the two drugs.

\section{UV-Vis And FL Spectra}

The UV spectra of the co-assembled HCPT/Ce6 NRs (Figure S1A) revealed the typical UV-vis absorbance peaks from both HCPT (384 nm) and Ce6 (405 and $652 \mathrm{~nm}$ ), together with an elevated overall baseline. Moreover, the co-assembly of HCPT with Ce6 resulted in a palpably broader and red-shifted Soret band of $\mathrm{Ce} 6$, along with an absorbance peak red shift of HCPT, indicating that the porphyrin core of $\mathrm{Ce} 6$ had interacted with the aromatic ring of HCPT via $\pi-\pi$ stacking and hydrophobic interactions. ${ }^{45}$

The fluorescence emission intensity of HCPT and Ce6 inside the HCPT/Ce6 NRs were significantly 

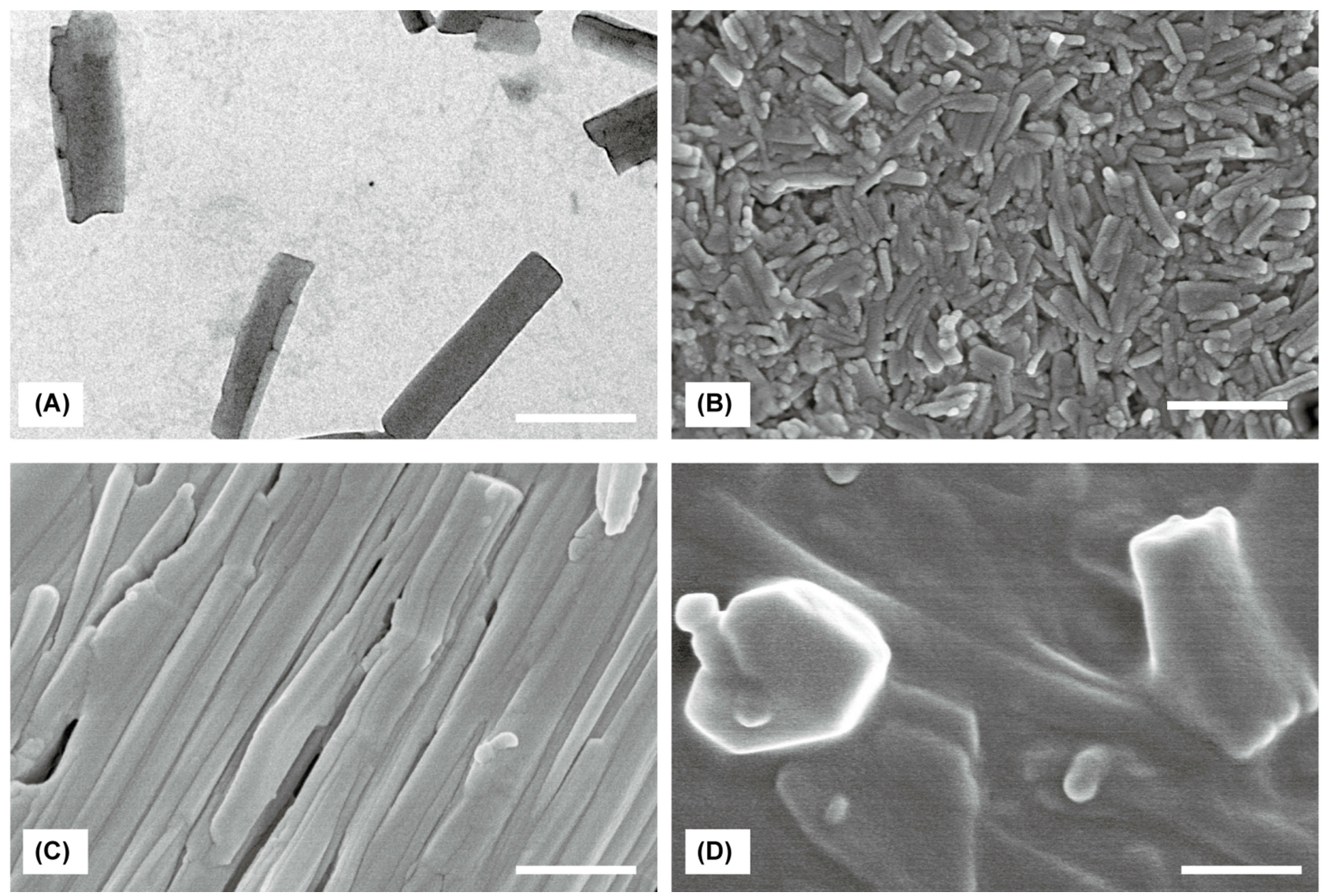

Figure 4 TEM images of (A) HCPT/Ce6 NRs (molar ratio HCPT: Ce6=3:I). SEM images of (B) HCPT/Ce6 NRs, (C) free HCPT powder and (D) free Ce6 powder. Scale bar: $200 \mathrm{~nm}$.

Abbreviations: HCPT, 10-hydroxycamptothecine; Ce6, Chlorin e6; NRs, nanorods; TEM, transmission electron microscope; SEM, scanning electron microscope.

reduced compared with monomeric HCPT and Ce6 (Figure S1B). Fluorescence quenching of the NRs might ascribe to the Dexter-type exciton migration between stacked HCPT or Ce6 molecules, which could be explained by the absorbance red shift of their UV spectrum $(384 \rightarrow 402 \mathrm{~nm}$ and $652 \rightarrow 670 \mathrm{~nm}) .{ }^{46}$ The results of UV and FL demonstrated that hydrophobic and $\pi-\pi$ stacking interactions might be one of the major driving forces for the formation of HCPT/Ce6 NRs, which was in accordance with the ITC results.

\section{Fourier-Transform Infrared Spectroscopy (FT-IR)}

The spectra of raw HCPT, raw Ce6, physical mixture of HCPT and Ce6, and HCPT/Ce6 NRs were shown in Figure 5A. The intensive and broad absorption bands of various samples were focused at $3351-3479 \mathrm{~cm}^{-1}$ due to the stretching of hydroxyl groups with the addition of adsorbed water molecule. In the spectrum of raw HCPT (Figure 5Ab), the characteristic peaks were observed at $1723 \mathrm{~cm}^{-1}$ ( $\mathrm{C}=\mathrm{O}$ in lactone ring), $1654 \mathrm{~cm}^{-1}$ (acylamino group), $1595 \mathrm{~cm}^{-1}$ (aromatic frame), and $1503 \mathrm{~cm}^{-1}$ (aromatic ring). The spectrum of raw $\mathrm{Ce} 6$ (Figure 5Aa) presented characteristic peaks at $2923 \mathrm{~cm}^{-1}$ and $2853 \mathrm{~cm}^{-1}$ (C-H in methyl or methylene group), $1715 \mathrm{~cm}^{-1}(\mathrm{C}=\mathrm{O}$ in carboxyl $), 1601 \mathrm{~cm}^{-1}$ $(\mathrm{C}=\mathrm{C}), 1510 \mathrm{~cm}^{-1}$ (aromatic frame), and $1448 \mathrm{~cm}^{-1}$ (aromatic frame). The characteristic bands in the IR spectra of the physical mixture (Figure 5Ac) almost corresponded to that of HCPT and Ce6 raw materials, while obvious weak peaks in the aromatic ring $\left(1596 \mathrm{~cm}^{-1}\right.$ $1400 \mathrm{~cm}^{-1}$ ) and disappear of some sharp absorption peaks in fingerprint region $\left(1350 \mathrm{~cm}^{-1}-500 \mathrm{~cm}^{-1}\right)$ were observed in HCPT/Ce6 NRs pattern (Figure 5Ad), indicating possible molecular interactions between the two molecules. Furthermore, the peak of raw HCPT at $1723 \mathrm{~cm}^{-1}$, assigning to the stretching vibration of $\mathrm{C}=\mathrm{O}$ in the terminal lactone ring, existed in the HCPT/ Ce6 NRs spectrum as well, certifying the reservation of lactone form for HCPT in the NRs. ${ }^{47}$ 

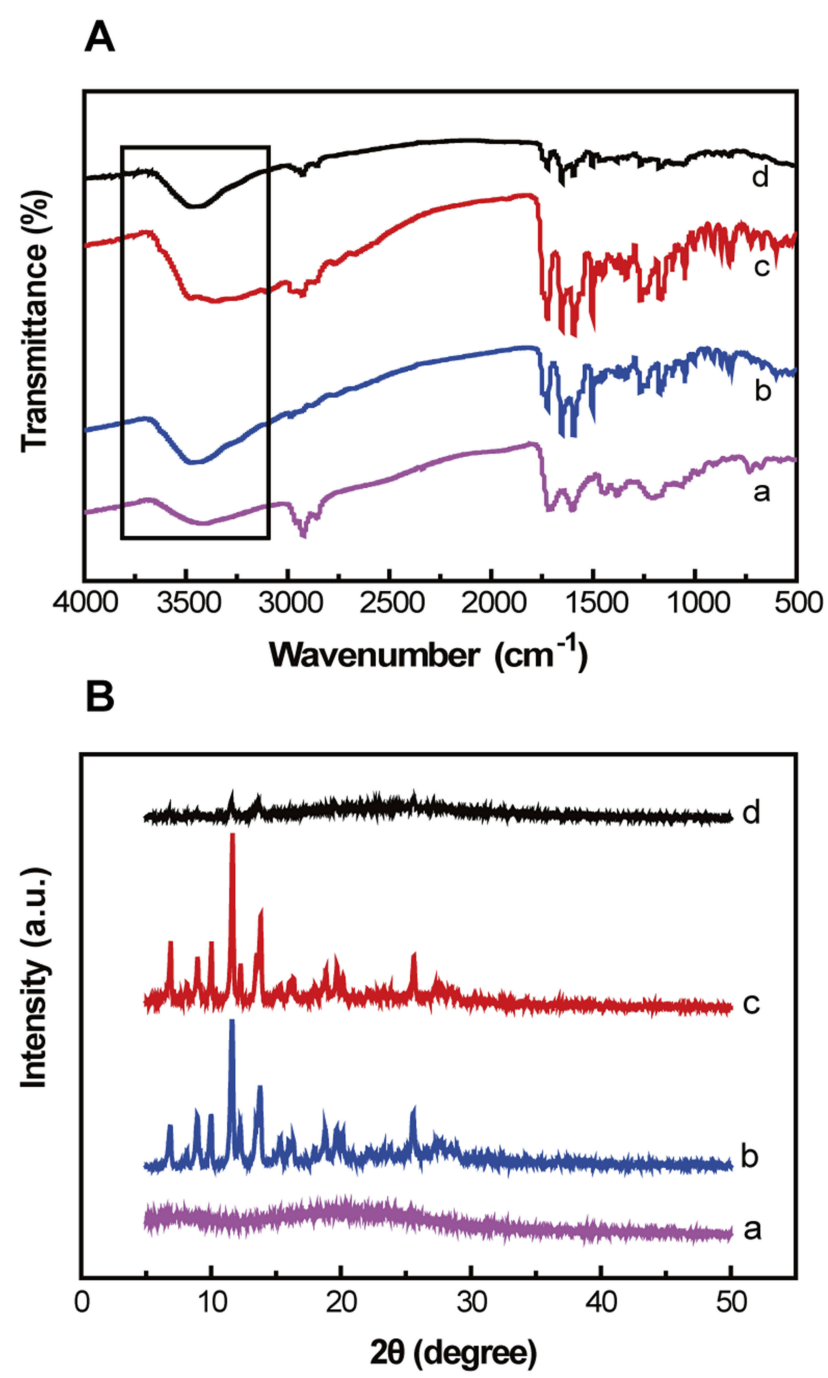

Figure 5 (A) FT-IR spectra and (B) XRD patterns of (a) raw Ce6, (b) raw HCPT, (c) physical mixture of HCPT and Ce6, and (d) HCPT/Ce6 nanorods.

Abbreviations: HCPT, 10-hydroxycamptothecine; Ce6, Chlorin e6; FT-IR, fouriertransform infrared; $X R D$, powder $X$-ray diffraction

\section{Powder X-Ray Diffraction (XRD)}

Figure $5 \mathrm{~B}$ displayed the XRD patterns of pure HCPT powder, pure $\mathrm{Ce} 6$ powder, physical mixture of HCPT and $\mathrm{Ce} 6$, and HCPT/Ce6 NRs. The XRD pattern of pure HCPT (Figure $5 \mathrm{Bb}$ ) exhibited typical high-energy diffraction peaks $\left(2 \theta\right.$ scale of $6.76^{\circ}, 8.85^{\circ}, 11.54^{\circ}, 13.46^{\circ}$, $19.59^{\circ}$, and $25.55^{\circ}$ ), indicating the crystallographic texture of $\mathrm{HCPT}^{48}$ Besides, no characteristic intense peaks but a very smooth curve of pure Ce6 (Figure 5Ba) was observed. The pattern of physical mixture (Figure 5Bc) and NRs (Figure 5Bd) was well identified with that of HCPT raw material for the preservation of its characteristic peaks at $2 \theta$ from $6^{\circ}$ to $30^{\circ}$, suggesting that $\mathrm{HCPT}$ existed as crystalline form inside NRs. The obvious weak peaks indicated the apparent decrease in crystallinity of HCPT in NRs, which might be due to the possible interaction between HCPT and Ce6 as well.

\section{Stability Study}

For storage stability, HCPT/Ce6 NRs were stored at $4{ }^{\circ} \mathrm{C}$ for 21 days, and the particle size as well as zeta potential of the NRs were monitored by DLS. Although the average data fluctuated slightly, no obvious difference was observed in both size (Figure 3C) and zeta potential (Figure 3D) compared with the initial value at 0 day. These results indicated that HCPT/Ce6 NRs had good storage stability in the aqueous state, which was possibly due to the associative forces between the two drugs, including $\pi-\pi$ stacking, and hydrophobic interactions. ${ }^{11}$

For the lyophilized stability, HCPT/Ce6 NRs were firstly lyophilized with different cryoprotectants in a proportion of $1 \%(\mathrm{w} / \mathrm{v})$ and reconstructed. The DLS results (Figure S2A) suggested that the NRs with trehalose as cryoprotectant presented the smallest particle size of $174.5 \pm 2.0 \mathrm{~nm}$ in contrast with other cryoprotectants. As a result, the NRs with various proportions of trehalose were further studied and it was found that the NRs with $2 \%$ trehalose presented the smallest particle size of 128.7 $\pm 1.2 \mathrm{~nm}$ compared with other proportions (Figure S2B). Besides, during storage for up to 21 days at room temperature, the lyophilized NRs with $2 \%$ trehalose presented similar particle size of approximately $150 \mathrm{~nm}$ (Figure S2C) with primal NRs, which revealed favorable stability of the freeze-dried NRs.

\section{In Vitro Drug Release Behavior}

The in vitro release performance of HCPT/Ce6 NRs was studied and compared with HCPT injection and Ce6 injection. As shown in Figure 6A, there was no statistical difference in the release property between HCPT injection and $\mathrm{Ce} 6$ injection, and both drugs showed burst release of up to $90 \%$ within the initial $2 \mathrm{hrs}$ and almost $100 \%$ at 4 hrs, exhibiting a complete passive diffusion release behavior. On the contrary, both drugs (HCPT and Ce6) in the NRs displayed slower release rate compared with the injection. For instance, only small amount of HCPT (around 20\%) in dual-drug NRs released at $2 \mathrm{hrs,} \mathrm{together}$ with above $80 \%$ released at 12 hrs, followed by significantly extended release until 24 hrs. Besides, the release rate of $\mathrm{Ce} 6$ from the NRs was a little faster than that of HCPT within the initial 4 hrs and there was almost $80 \%$ of Ce6 released within 24 hrs. The relatively sustained- 


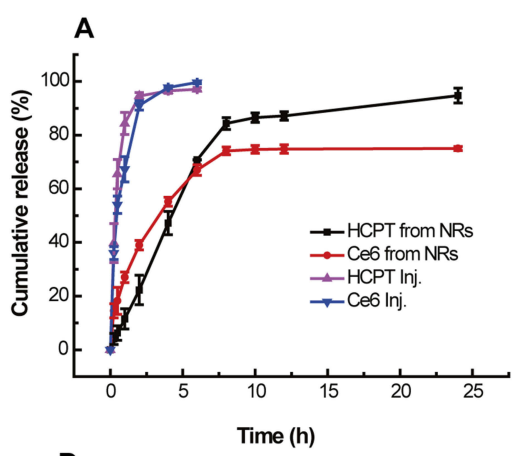

D

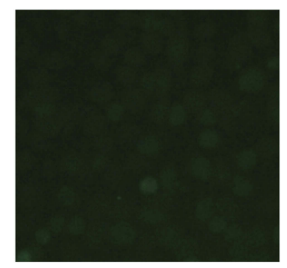

PBS
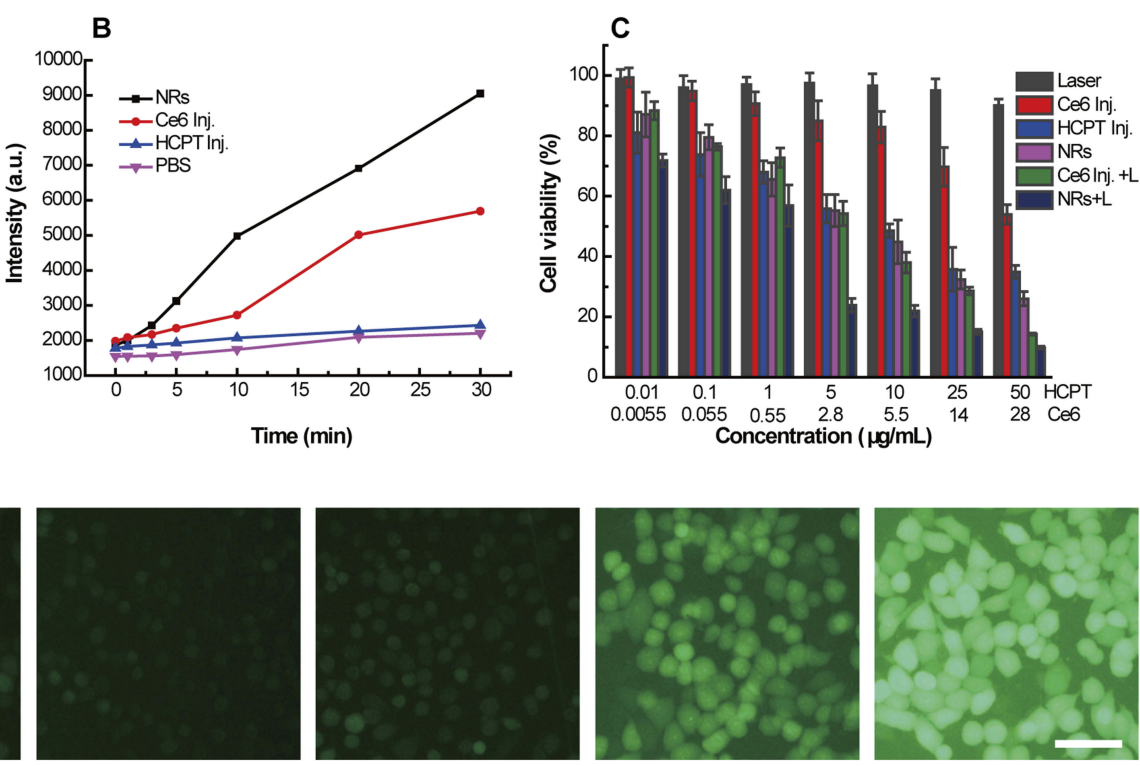

HCPT Inj.

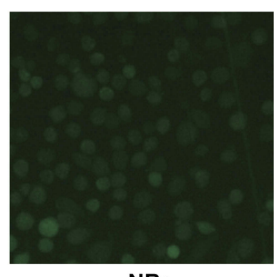

NRs

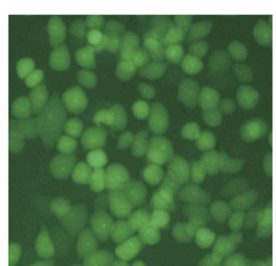

Ce6 Inj. +L

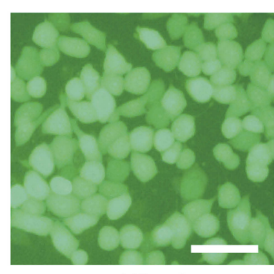

NRs+L

Figure 6 (A) The in vitro cumulative drug release curves of HCPT and Ce6 from NRs and injection. (B) Fluorescence intensity variation of SOSG-EP ( $E_{\mathrm{x}}=498 \mathrm{~nm}, \mathrm{E}_{\mathrm{m}}=525.8$ $\mathrm{nm}$ ) via a fluorescence spectrophotometer at the characteristic peak against the irradiation time. (C) In vitro cell viability of $4 \mathrm{TI}$ cells after incubation with various formulations with or without laser irradiation ( $\lambda=660 \mathrm{~nm}, 5 \mathrm{~mW}, 2$ mins). (D) Intracellular ROS level evaluation using DCFH-DA. Fluorescence microscope images of 4TI cells after incubation with different formulations followed with or without laser irradiation ( $\lambda=660 \mathrm{~nm}, 5 \mathrm{~mW}, 5 \mathrm{mins})$. Scale bar: $100 \mu \mathrm{m}$.

Abbreviations: HCPT, 10-hydroxycamptothecine; Ce6, Chlorin e6; NRs, nanorods; Inj., injection; L, laser; PBS, phosphate buffer saline.

release of HCPT/Ce6 NRs should be attributed to the slow diffusion of HCPT and Ce6 from the NRs rather than simple penetration of drug molecules through the dialysis membrane, ${ }^{49}$ and the existing $\pi-\pi$ interaction and electrostatic interaction between HCPT and Ce6 in the NRs. ${ }^{46}$ The release behavior of HCPT and $\mathrm{Ce} 6$ from the NRs was almost synchronous, which might be beneficial to exert the synergetic chemotherapy and photodynamic therapy (PDT) efficacy of the NRs.

\section{In Vitro Cytotoxicity Effect}

To further examine the in vitro antitumor efficiency of the dual-functional NRs, the cytotoxicity of various formulations (HCPT injection, Ce6 injection, and HCPT/ Ce6 NRs) exposure to laser irradiation (or not) against 4T1 breast cancer cell lines was analyzed by MTT assay with concentration of HCPT ranging from 0.01 to $50 \mu \mathrm{g} /$ $\mathrm{mL}$ and Ce6 ranging from 0.0055 to $28 \mu \mathrm{g} / \mathrm{mL}$. As shown in Figure 6C, the mere laser irradiation hardly showed any cytotoxicity against 4T1 cells (inhibition $<5 \%$ ), while the groups treated with HCPT injection and HCPT/Ce6 NRs (without laser) revealed a relatively medium tumor cells growth suppression. It was also obvious that $\mathrm{Ce} 6$ injection without laser irradiation exhibited minor toxicity, whereas an obviously reduced viability of cells was observed when treated with Ce6 injection in the presence of laser irradiation. However, the NRs with laser irradiation displayed significantly decreased cell viability against the increasing dosage in comparison with other formulations as expected, demonstrating the effectively combined anticancer efficiency of chemotherapy (HCPT) and PDT (Ce6). In addition, the half-maximal inhibitory concentration $\left(\mathrm{IC}_{50}\right)$ (Table S3) of HCPT/Ce6 NRs (+Laser) was measured as $0.41 \mu \mathrm{g} /$ $\mathrm{mL}$, which was about 16 -fold lower than that of free HCPT $(6.35 \mu \mathrm{g} / \mathrm{mL})$ and 7 -fold lower than that of free Ce6 $(2.71 \mu \mathrm{g} / \mathrm{mL})$. Besides, the CI values of the NRs at different concentrations calculated according to their MTT results were all smaller than 1 (Figure S3). All these results certified the synergistic antitumor efficacy of chemotherapy (HCPT) and PDT (Ce6).

\section{In Vitro Cellular Uptake}

The intracellular uptake of $\mathrm{Ce} 6$ in dual-functional HCPT/Ce6 NRs against 4T1 cells was surveyed with fluorescence microscope (FM), as Ce6 could exhibit obvious red fluorescence under irradiation. As shown in Figure 7D, strong red fluorescence was observed in the cytoplasm of most cells incubated with HCPT/Ce6 NRs for 4 hrs, verifying well intracellular release behavior and heightened internalization level of the NRs against 4T1 cells. Furthermore, the practical content 

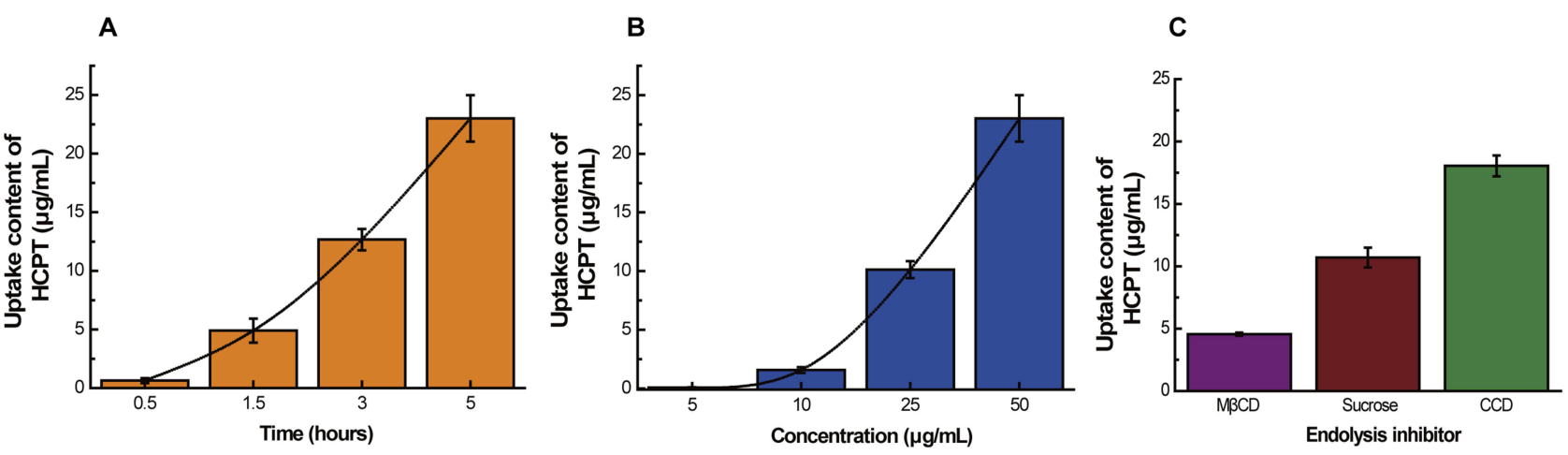

D

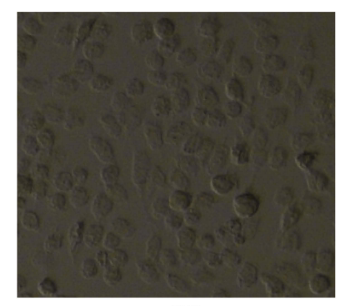

BF

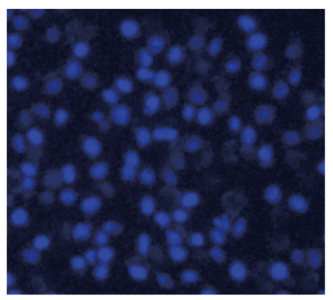

Hoechst

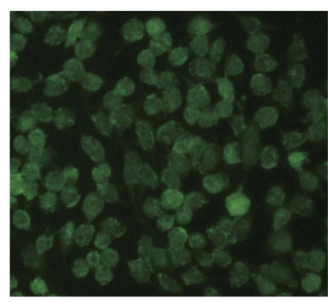

Lyso-Tracker

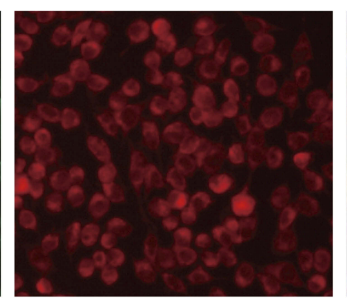

NRs

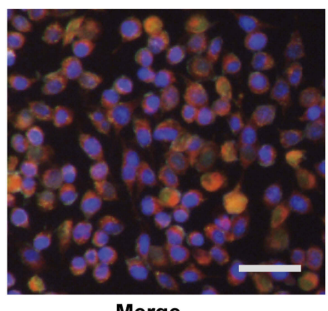

Merge

Figure 7 Intracellular uptake of HCPT/Ce6 NRs in 4TI cells assessed by HPLC-FLD system.

Notes: (A) The cellular uptake content of HCPT in $0.5,1.5,3$, and 5 hrs. (B) The cellular uptake content of HCPT in 5 , 10, 25, and 50 $\mu$ g/mL. (C) The cellular uptake content of HCPT after incubated with diverse endocytosis inhibitor Methyl- $\beta$-cyclodextrin (MBCD), Cytochalasin D (CCD) and Sucrose. (D) Fluorescence microscope images of $4 \mathrm{TI}$ cells after incubation with HCPT/Ce6 NRs (equal to $10 \mu \mathrm{g} / \mathrm{mL} \mathrm{Ce6)} \mathrm{for} 4 \mathrm{hrs}$. Ce6 (red fluorescence), Hoechst (blue fluorescence), and LysoTracker ${ }^{\circledR}$ Green (green fluorescence) were observed and represented NRs, nuclei, and lysosome, respectively. Scale bar: $100 \mu \mathrm{m}$.

Abbreviations: HCPT, 10-hydroxycamptothecine; Ce6, Chlorin e6; NRs, nanorods; BF, bright field; Hoechst, Hoechst 33258; LysoTracker, LysoTracker ${ }^{\circledR}$ Green.

of HCPT internalized by $4 \mathrm{~T} 1$ cells was quantified using HPLC-FLD. The uptake of HCPT by 4T1 cells increased from 0.08 to $23.01 \mu \mathrm{g} / \mathrm{mL}$ against the raised dosage of the NRs from 5 to $50 \mu \mathrm{g} / \mathrm{mL}$ (Figure 7B), indicating that the cell uptake of the NRs was a dosedependent process within certain range. In addition, the uptake of HCPT increased from 0.63 to $23.01 \mu \mathrm{g} / \mathrm{mL}$ against the enhanced incubation time from 0.5 to $5 \mathrm{hrs}$ (Figure 7A), indicating the time-dependent intracellular uptake behavior of the NRs within certain range. The cellular uptake results indicated that the dual-functional HCPT/Ce6 NRs were well uptake by the 4T1 cells and HCPT and Ce6 in the NRs were all successfully released, which might lead to a better therapeutic effect. Moreover, when incubated with diverse endocytosis inhibitor, such as methyl- $\beta$-cyclodextrin $(M \beta C D)$, cytochalasin D (CCD) and sucrose, the cellular uptake results of $\mathrm{HCPT} / \mathrm{Ce} 6 \mathrm{NRs}$ by $4 \mathrm{~T} 1$ cells were significantly different. As seen in Figure $7 \mathrm{C}, \mathrm{CCD}$ had the least influence on the cell uptake efficiency of the NRs, followed by sucrose and $M \beta C D$. According to the report, ${ }^{50}$ $\mathrm{M} \beta \mathrm{CD}$ inhibited caveolin-mediated endocytosis, sucrose was a clathrin-mediated endocytosis inhibitor, and CCD was a large-pinocytosis-mediated cellular uptake inhibitor. Therefore, the cellular uptake pathways of HCPT/Ce6 NRs were mainly large-pinocytosis and clathrin-mediated endocytosis.

\section{Determination Of Singlet Oxygen}

The quantum yield of ${ }^{1} \mathrm{O}_{2}$ by the NRs was detected using the fluorescent probe singlet oxygen sensor green (SOSG). Once SOSG reacts chemically with ${ }^{1} \mathrm{O}_{2}$, it will transfer to endoperoxide (SOSG-EP) with distinct fluorescence characteristics, which can be utilized to detect ${ }^{1} \mathrm{O}_{2}$ production. ${ }^{51}$ Figure $6 \mathrm{~B}$ showed the fluorescence intensity of SOSG with various formulations against the irradiation time. The fluorescence intensity of both PBS and HCPT injection changed slightly after exposure to laser irradiation for 30 mins, demonstrating that PBS or HCPT injection alone could not emerge ${ }^{1} \mathrm{O}_{2}$. It was rather remarkable that $\mathrm{Ce} 6$ injection revealed a relatively slower production quotiety of ${ }^{1} \mathrm{O}_{2}$, while HCPT/ Ce6 NRs preserved an obviously rapid ${ }^{1} \mathrm{O}_{2}$ generation rate. Besides, the fluorescence intensity of SOSG-EP 
showed a time-dependent enhancement in the presence of NRs upon specified laser irradiation time (Figure S4B), which could possibly be explained as Ce6 molecules assembled with each other and easily caused fluorescence quenching in the injection, but when aggregated on the surface of the NRs and interacted with HCPT molecules, higher dispersion and stability could be acquired, which heightened the ${ }^{1} \mathrm{O}_{2}$ yield productivity of $\mathrm{Ce} 6 .{ }^{15}$

To evaluate the intracellular ROS generation capacity of HCPT/Ce6 NRs, DCFH-DA was chosen as cellular fluorescent probe. DCFH-DA itself has no fluorescence, but when uptake by the cells, it can be hydrolyzed to DCFH with intracellular esterase. And then, with the existence of ROS, the DCFH is ultimately oxidized to DCF, which can glow highly green fluorescence and be utilized to detect the intracellular ROS level. ${ }^{52}$ As shown in Figure S4A, after incubation for $4 \mathrm{hrs}$ and exposure to laser irradiation for $2 \mathrm{mins}$, the fluorescence intensity of HCPT injection groups changed inapparently against the increased concentration, whereas the Ce6 injection groups revealed a slowly enhanced profile of fluorescence intensity. As expected, the HCPT/Ce6 NRs preserved an obviously raised plot, indicating that the PDT effect of the NRs was superior to that of Ce6 injection. Conclusively, it was speculated that the NRs could be efficiently internalized by tumor cells and the drugs could be well released in tumor microenvironment. ${ }^{53}$

The intracellular ROS production capacity of the NRs was further affirmed by fluorescence micrograph observation. Cells were treated with blank medium, HCPT injection, Ce6 injection, and HCPT/Ce6 NRs, with or without laser irradiation. All the cells exhibited negligible DCFH-DA fluorescence without laser irradiation, whereas cells treated with NRs generated potent fluorescence signal of ROS upon laser exposure compared with that of Ce6 injection (Figure 6D). The pronounced ROS generation capacity of HCPT/Ce6 NRs should be attributed to the stronger cellular uptake together with higher dispersion and stability of $\mathrm{Ce} 6$ molecules in the NRs, which induced oxidase activities and, consequently, ROS generation. ${ }^{54,55}$ In addition, cells treated with $\mathrm{HCPT} / \mathrm{Ce} 6 \mathrm{NRs}$ displayed powerful time and dose-dependent DCFH-DA fluorescence signal at different time intervals (Figure S5A) or various $\mathrm{Ce} 6$ concentrations (Figure S5B). Therefore, HCPT/Ce6 NRs exhibited better prolificacy of ${ }^{1} \mathrm{O}_{2}$ and could be applied as a promising candidate for PDT treatment in vivo.

\section{In Vivo Therapeutic Efficacy}

Inspired by the remarkable in vitro chemo-phototherapy antitumor efficacy and ROS production capacity of the dual-functional NRs, we further measured the in vivo antitumor efficacy of HCPT/Ce6 NRs with 4T1 tumorbearing mouse models. As shown in Figure 8A, the tumor volume of the control NS group increased rapidly and eventually reached to $1556.28 \pm 282.97 \mathrm{~mm}^{3}$ at the end (10 days) of the experiment, while the groups treated with HCPT injection, Ce6 injection (with laser) and the mixture of HCPT and Ce6 injection (with laser) exhibited a relatively medium tumor suppression. As expected, mice treated with HCPT/Ce6 NRs under laser irradiation performed a dramatic tumor suppression than that of HCPT/Ce6 NRs (without laser) and other injection groups, which was further attested by visual observation of the collected tumor tissues for each group (Figure 8D). The excellent inhibition efficacy on tumor growth of the NRs containing photosensitizer and chemotherapeutic drug might be arisen from the synergistic effect of the combined chemo-phototherapy provided by the two drugs.

Tumor inhibition rate (TIR\%) was also calculated based on the average tumor weights in each group (Figure 8C). Compared with the saline control, the HCPT/Ce6 NR (with laser) was $86.72 \pm 0.97 \%$, which was significantly higher than that of HCPT injection $(49.44 \pm 12.77 \%)$, Ce6 injection under laser irradiation $(40.00 \pm 24.67 \%)$, HCPT and Ce6 injection mixture with laser $(50.63 \pm 18.01 \%)$ and NRs without laser (69.87 $\pm 7.86 \%$ ). It was worth noting that the combined chemo-phototherapy of HCPT/Ce6 NRs under laser irradiation was more cytotoxic to tumor cells than the photodynamic therapy or the chemotherapy treatment alone owing to the synergistic effect. The intensive tumor inhibition rate of the NRs might be attributed to the considerably increased cell uptake and higher accumulation in the tumor through EPR effect. ${ }^{56}$

In order to further confirm the therapeutic effects, hematoxylin-eosin staining (H\&E) was introduced to observe the morphology of tumor cells after treated with various formulations. As shown in Figure 8E, the tumor cells treated with $\mathrm{HCPT} / \mathrm{Ce} 6 \mathrm{NRs}$ under laser irradiation exhibited severe damage and irregular shape with shrinking nucleus, while tumor cells in other groups had no 
A

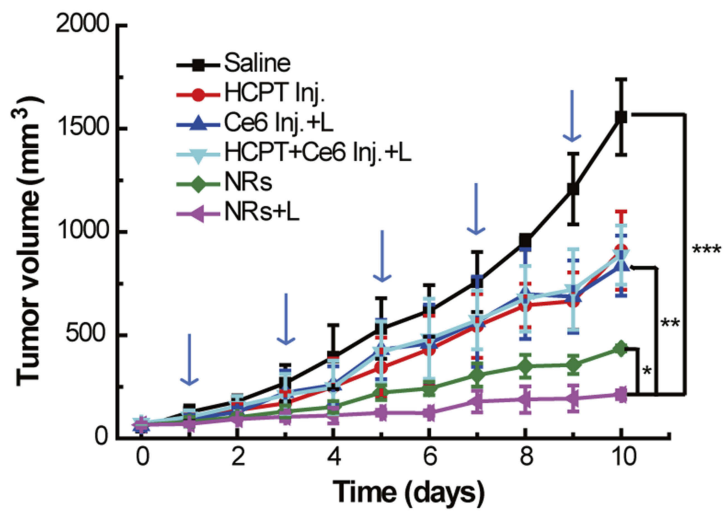

C

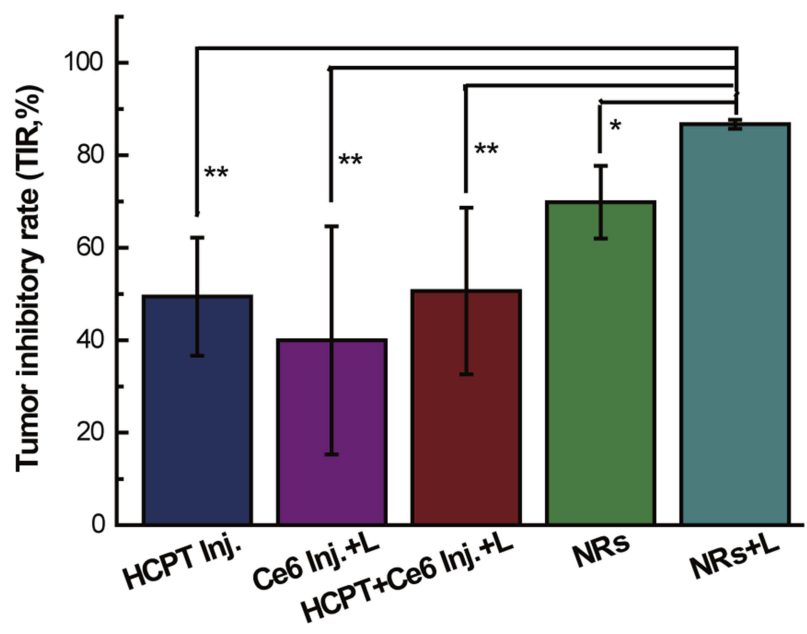

E

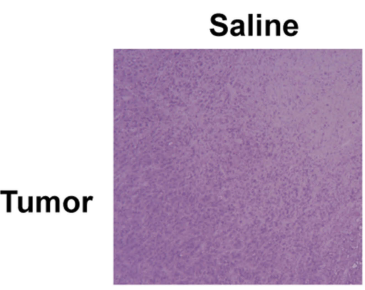

HCPT Inj.

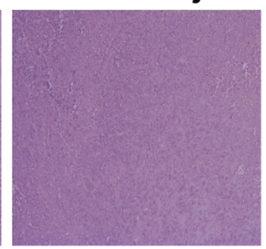

Ce6 Inj.+L

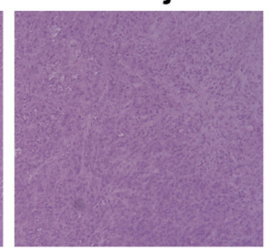

B

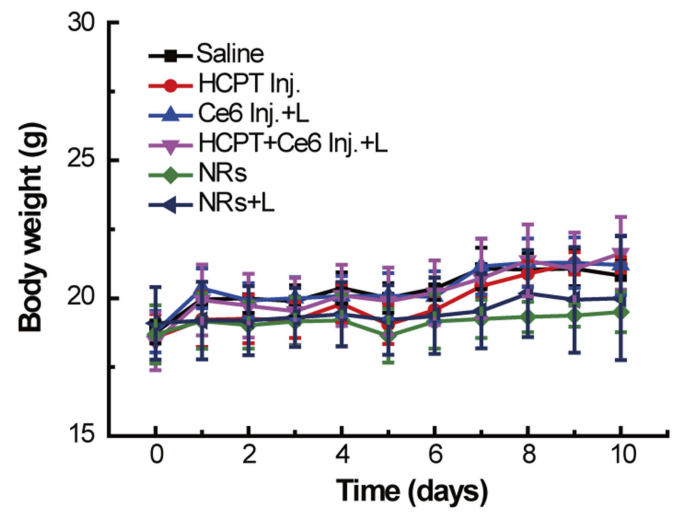

D
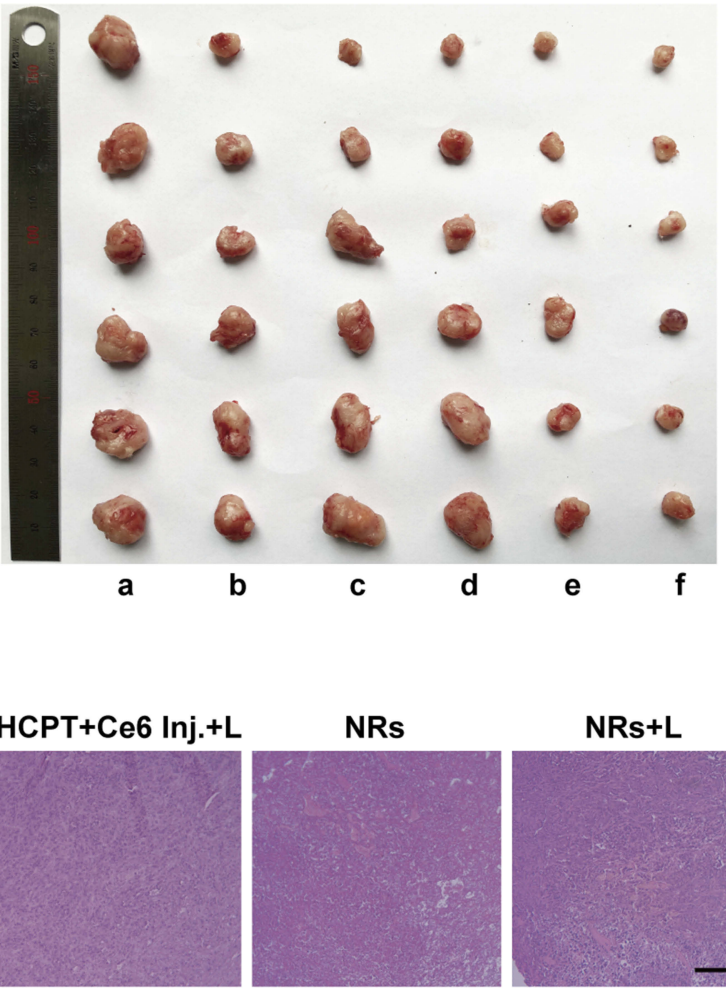

NRs

NRs+L
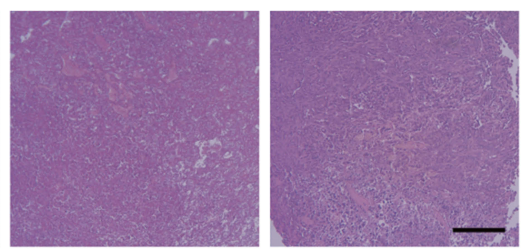

Figure 8 (A) Suppression of tumor growth after various treatments: intravenous injection of different formulations with or without laser irradiation ( $n=6)$. ${ }^{*}<0.05$, NRs versus NRs+L; **p < 0.0I, Ce6 Inj.+L, HCPT Inj. or HCPT+Ce6 Inj. $+\mathrm{L}$ versus NRs $+\mathrm{L}$; ***P < $0.00 \mathrm{I}$, saline versus NRs $+\mathrm{L}$. (B) Body weight changes of tumor-bearing mice during the treatment $(n=6)$. (C) Tumor inhibition rate $(T I R \%)$ based on the average tumor weights in each group $(n=6)$. ${ }^{*} P<0.05, N R s$ versus NRs $+L ; * * p<0.0 I$, Ce6 Inj. $+L$, HCPT Inj. or HCPT+Ce6 Inj.+L versus NRs+L. (D) Images of the excised 4TI tumor tissues after various treatments at 10 days post implantation. (a) Saline; (b) HCPT Inj.; (c) Ce6 Inj.+L; (d) HCPT+Ce6 Inj.+L; (e) NRs; (f) NRs+L. (E) Hematoxylin and eosin (H\&E) stained histological images of tumor tissues from different groups of 4TI tumorbearing mice after treated with various formulations. Scale bar: $100 \mu \mathrm{m}$.

Abbreviations: Inj., injection; L, laser; NRs, nanorods; HCPT, I0-hydroxycamptothecine; Ce6, Chlorin e6.

apparent damage or other inflammatory and immune response. These H\&E results further demonstrated the synergistic therapeutic effect of the NRs.

\section{In Vivo Study Of Toxicity}

As the in vivo latent toxicity has always been a problem in the development of novel formulations, ${ }^{57}$ the in vivo safety of the NRs was evaluated. Firstly, the body weights of all mice (Figure 8B) were monitored every day and revealed no obvious differences among groups during the whole experimental period. Furthermore, H\&E staining analysis of the collected main organs (heart, liver, spleen, kidney, and lung) showed no significant cell damage and morphological changes among groups (Figure 9). 


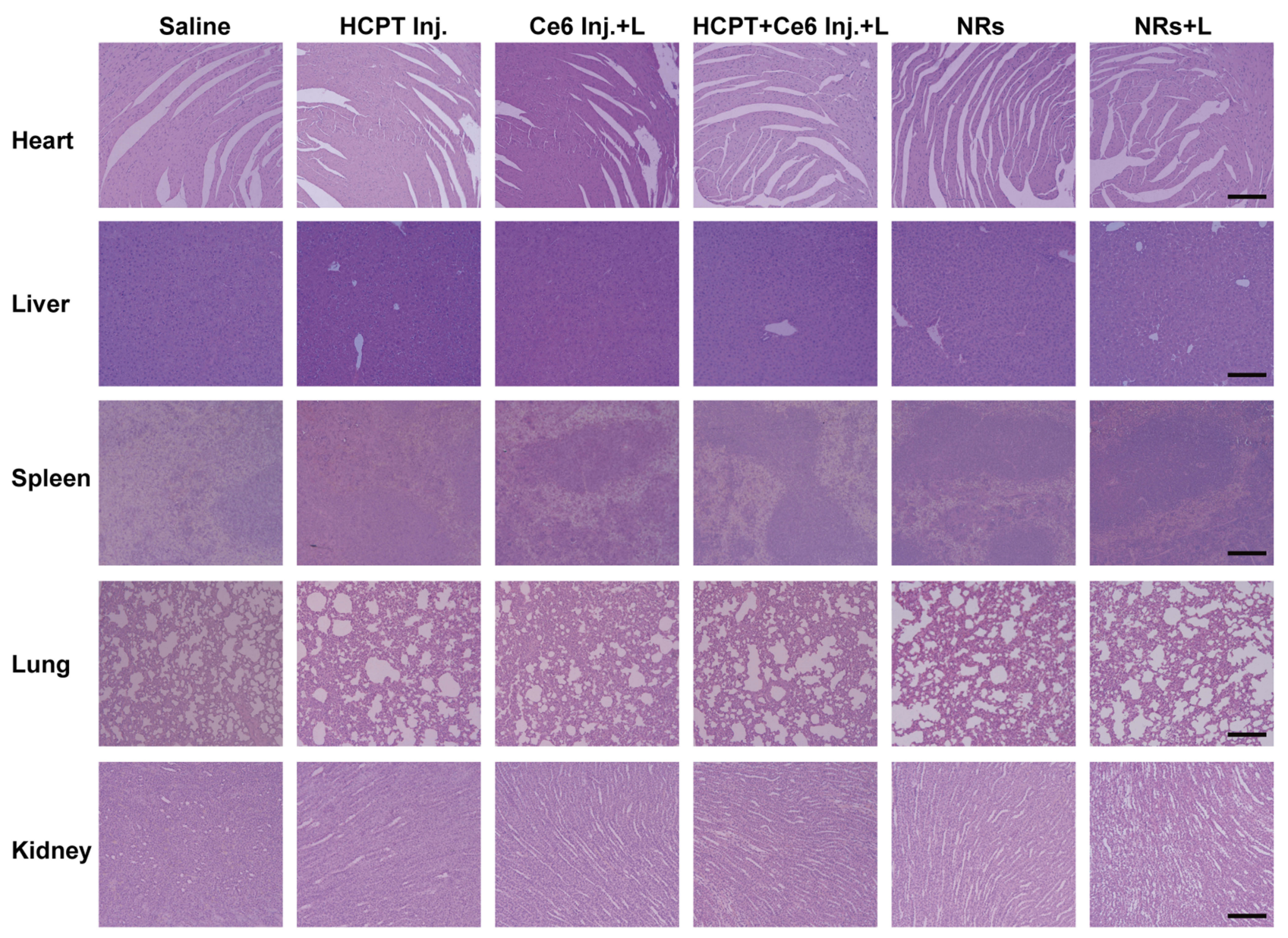

Figure $9 \mathrm{H \& E}$ stained histological images obtained from main organs (heart, liver, spleen, lung, and kidney) of 4TI tumor-bearing mice after treated with various formulations. Scale bar: $100 \mu \mathrm{m}$.

Abbreviations: Inj., injection; L, laser; NRs, nanorods; HCPT, I0-hydroxycamptothecine; Ce6, Chlorin e6.

Moreover, the aspartate aminotransferase (AST) and alanine aminotransferase (ALT) as well as blood urea nitrogen (BUN) and creatinine (CRE), which were the most common parameters to evaluate the function of liver and kidney, ${ }^{58}$ were analyzed for evaluating the safety of dual- functional NRs. The results (Table 3) showed that there was no significant difference in AST, ALT, BUN, and CRE levels of the NRs group when compared to the control group ( $p>0.05$ ), indicating the HCPT/Ce6 NRs had no obvious hepatic or renal toxicity. All the in vivo toxicity

Table 3 Plasma Biochemical Levels Of 4TI Bearing BALB/c Mice After Treatment With Different Formulations

\begin{tabular}{|l|l|l|l|l|}
\hline Group & $\begin{array}{l}\text { ALT } \\
(\mathbf{I U / L})\end{array}$ & $\begin{array}{l}\text { AST } \\
(\mathbf{I U / L})\end{array}$ & $\begin{array}{l}\text { BUN } \\
(\mathbf{m m o l} / \mathbf{L})\end{array}$ & $\begin{array}{l}\text { CRE } \\
(\boldsymbol{\mu m o l / L )})\end{array}$ \\
\hline Saline & $110.1 \pm 8.0$ & $112.5 \pm 5.7$ & $7.3 \pm 0.3$ & $47.1 \pm 4.3$ \\
HCPT Inj. & $106.8 \pm 9.3$ & $105.0 \pm 9.7$ & $7.2 \pm 0.5$ & $43.1 \pm 7.2$ \\
Ce6 Inj.+L & $104.6 \pm 12.7$ & $107.6 \pm 23.5$ & $7.1 \pm 0.6$ & $43.9 \pm 7.1$ \\
HCPT+Ce6 Inj.+L & $112.5 \pm 9.8$ & $113.4 \pm 11.2$ & $6.8 \pm 0.6$ & $44.0 \pm 6.8$ \\
NRs & $108.2 \pm 10.1$ & $115.4 \pm 11.5$ & $7.1 \pm 0.9$ & $48.3 \pm 6.8$ \\
NRs+L & $107.5 \pm 11.4$ & $113.9 \pm 18.3$ & $7.1 \pm 1.2$ & $45.8 \pm 5.7$ \\
\hline
\end{tabular}

Notes: There was no significant difference in the parameters (ALT, AST, BUN, and CRE) among different formulations when compared with the saline group (P > 0.05). All results are presented as mean $\pm S D(n=6)$.

Abbreviations: HCPT, I0-hydroxycamptothecine; Ce6, Chlorin e6; ALT, alanine transaminase; AST, aspartate transaminase; BUN, blood urea nitrogen; CRE, creatinine; Inj., injection; L, laser; NRs, nanorods. 
results indicated that $\mathrm{HCPT} / \mathrm{Ce} 6 \mathrm{NRs}$ were relatively safe without any immune response or inducing inflammation.

\section{Conclusion}

The novel carrier-free dual-functional HCPT/Ce6 NRs were developed with pure drugs (HCPT and Ce6) by a simple self-assembly procedure. HCPT and Ce6 molecules tended to form co-assembled NRs through the hydrophobic interactions (the $\pi-\pi$ conjugation of the ring structure) and electrostatic interactions. The as-prepared HCPT/Ce6 NRs exhibited uniform morphology with excellent stability both in aqueous solution and freeze-dried state and relatively slower release rate compared with the injections. Furthermore, the obtained HCPT/Ce6 NRs not only showed considerable cell uptake efficiency but also exhibited significant extraor intracellular ROS producing capacity in the presence of laser irradiation. In addition, the in vitro and in vivo antitumor studies showed that the dual-functional HCPT/Ce6 NRs exhibited a substantially synergistic antitumor efficacy compared with mono-chemotherapy or mono-photodynamic therapy. Therefore, the rationale of this facile and effective strategy for fabricating the carrier-free dual-functional NRs might open an alternative avenue and give inspiration for the development of combinational antitumor therapy in a synergistic way.

\section{Acknowledgment}

This work was financially supported by the National Science and Technology Major Project (no. 2017ZX09201-003), the National Nature Science Foundation of China (no. 21473085), the Natural Science Foundation of Shandong Province of China (no. ZR2017BH065, no. ZR2016CL14), Project of Shandong Province Higher Educational Science and Technology Program (no. J17KA234), the Open Project of Shandong Collaborative Innovation Center for Antibody Drugs (no. CIC-AD1831), the Doctoral Starting up Foundation of Liaocheng University (no. 318051633), and Tai-Shan Scholar Research Fund of Shandong Province of China. The above work was also technically supported by Engineering Research Center for Nanomedicine and Drug Delivery Systems.

\section{Disclosure}

The authors declare no conflicts of interest in this study.

\section{References}

1. Bray F, Ferlay J, Soerjomataram I, Siegel RL, Torre LA, Jemal A. Global cancer statistics 2018: GLOBOCAN estimates of incidence and mortality worldwide for 36 cancers in 185 countries. CA Cancer $J$ Clin. 2018;68(6):394-424. doi:10.3322/caac.21492

2. Wang L, Wang Y, Wong JI, Palacios T, Kong J, Yang HY. Functionalized $\operatorname{MoS}(2)$ nanosheet-based field-effect biosensor for label-free sensitive detection of cancer marker proteins in solution. Small. 2014;10(6):1101-1105. doi:10.1002/smll.201302081

3. Hou W, Zhao X, Qian X, et al. pH-sensitive self-assembling nanoparticles for tumor near-infrared fluorescence imaging and chemo-photodynamic combination therapy. Nanoscale. 2016;8(1):104-116. doi:10.1039/c5nr06842h

4. Cai Y, Shen H, Zhan J, et al. Supramolecular "trojan horse" for nuclear delivery of dual anticancer drugs. J Am Chem Soc. 2017;139(8):28762879. doi: $10.1021 /$ jacs.6b12322

5. Li Y, Lin J, Huang Y, et al. Self-targeted, shape-assisted, and controlled-release self-delivery nanodrug for synergistic targeting/anticancer effect of cytoplasm and nucleus of cancer cells. ACS Appl Mater Interfaces. 2015;7(46):25553-25559. doi:10.1021/acsami.5b07348

6. Zhao C, Cao W, Zheng $\mathrm{H}$, et al. Acid-responsive nanoparticles as a novel oxidative stress-inducing anticancer therapeutic agent for colon cancer. Int J Nanomedicine. 2019;14:1597-1618. doi:10.2147/IJN. S189923

7. Zu Y, Meng L, Zhao X, et al. Preparation of 10-hydroxycamptothecinloaded glycyrrhizic acid-conjugated bovine serum albumin nanoparticles for hepatocellular carcinoma-targeted drug delivery. Int $J$ Nanomedicine. 2013;8:1207-1222. doi:10.2147/IJN.S40493

8. Li W, Zhang X, Hao X, Jie J, Tian B, Zhang X. Shape design of high drug payload nanoparticles for more effective cancer therapy. Chem Commun. 2013;49(93):10989-10991. doi:10.1039/c3cc46718j

9. Zhang L, Hu Y, Jiang X, Yang C, Lu W, Yang YH. Camptothecin derivative-loaded poly(caprolactone-co-lactide)-b-PEG-b-poly(caprolactone-co-lactide) nanoparticles and their biodistribution in mice. $J$ Control Release. 2004;96(1):135-148. doi:10.1016/j.jconrel.2004.0 1.010

10. Yang J, Teng Y, Fu Y, Zhang C. Chlorins e6 loaded silica nanoparticles coated with gastric cancer cell membrane for tumor specific photodynamic therapy of gastric cancer. Int $J$ Nanomedicine. 2019;14:5061-5071. doi:10.2147/IJN.S202910

11. Zhang R, Xing R, Jiao T, et al. Carrier-free, chemophotodynamic dual nanodrugs via self-assembly for synergistic antitumor therapy. ACS Appl Mater Interfaces. 2016;8(21):13262-13269. doi:10.1021/ acsami.6b02416

12. Hong EJ, Choi DG, Shim MS. Targeted and effective photodynamic therapy for cancer using functionalized nanomaterials. Acta Pharm Sin B. 2016;6(4):297-307. doi:10.1016/j.apsb.2016.01.007

13. Zhu X, Wang H, Zheng L, et al. Upconversion nanoparticle-mediated photodynamic therapy induces THP-1 macrophage apoptosis via ROS bursts and activation of the mitochondrial caspase pathway. Int J Nanomedicine. 2015;10:3719-3736. doi:10.2147/IJN.S82162

14. Ding YF, Li S, Liang L, et al. Highly biocompatible chlorin e6loaded chitosan nanoparticles for improved photodynamic cancer therapy. ACS Appl Mater Interfaces. 2018;10(12):9980-9987. doi:10.1021/acsami.8b01522

15. Wen Y, Zhang W, Gong N, et al. Carrier-free, self-assembled pure drug nanorods composed of 10-hydroxycamptothecin and chlorin e6 for combinatorial chemo-photodynamic antitumor therapy. In Vivo. Nanoscale. 2017;9(38):14347-14356. doi:10.1039/C7NR03129G

16. Liao L, Liu J, Dreaden EC, et al. A convergent synthetic platform for single-nanoparticle combination cancer therapy: ratiometric loading and controlled release of cisplatin, doxorubicin, and camptothecin. $J$ Am Chem Soc. 2014;136(16):5896-5899. doi:10.1021/ja502011g 
17. Qi Z, Shi J, Zhang Z, Cao Y, Li J, Cao S. PEGylated graphene oxidecapped gold nanorods/silica nanoparticles as multifunctional drug delivery platform with enhanced near-infrared responsiveness. Mate Sci Eng C. 2019;104:109889. doi:10.1016/j.msec.2019.109889

18. Song Z, Liu Y, Shi J, et al. Hydroxyapatite/mesoporous silica coated gold nanorods with improved degradability as a multi-responsive drug delivery platform. Mate Sci Eng C. 2018;83:90-98. doi:10.1016/j.msec.2017.11.012

19. Li Y, Liu G, Ma J, et al. Chemotherapeutic drug-photothermal agent co-self-assembling nanoparticles for near-infrared fluorescence and photoacoustic dual-modal imaging-guided chemo-photothermal synergistic therapy. $J$ Control Release. 2017;258:95-107. doi:10.1016/j.jconrel.2017.05.011

20. Qin SY, Zhang AQ, Cheng SX, Rong L, Zhang XZ. Drug selfdelivery systems for cancer therapy. Biomaterials. 2017;112:234247. doi:10.1016/j.biomaterials.2016.10.016

21. Mou Q, Ma Y, Zhu X, Yan D. A small molecule nanodrug consisting of amphiphilic targeting ligand-chemotherapy drug conjugate for targeted cancer therapy. $J$ Control Release. 2016;230:34-44. doi:10.1016/j.jconrel.2016.03.037

22. Zhang J, Liang YC, Lin X, et al. Self-monitoring and self-delivery of photosensitizer-doped nanoparticles for highly effective combination cancer therapy in vitro and in vivo. ACS Nano. 2015;9(10):97419756. doi:10.1021/acsnano.5b02513

23. Zhao R, Zheng G, Fan L, et al. Carrier-free nanodrug by co-assembly of chemotherapeutic agent and photosensitizer for cancer imaging and chemo-photo combination therapy. Acta Biomater. 2018;70:197210. doi:10.1016/j.actbio.2018.01.028

24. Basu A, Kumar GS. Interaction of the dietary pigment curcumin with hemoglobin: energetics of the complexation. Food Funct. 2014;5 (8):1949-1955. doi:10.1039/c4fo00295d

25. Hess B, Kutzner C, Van Der Spoel D, Lindahl E. GROMACS 4: algorithms for highly efficient, load-balanced, and scalable molecular simulation. J Chem Theory Comput. 2008;4(3):435-447. doi:10.1021/ct700301q

26. Abel S, Dupradeau FY, Marchi M. Molecular dynamics simulations of a characteristic DPC micelle in water. J Chem Theory Comput. 2012;8(11):4610-4623. doi:10.1021/ct3003207

27. Berendsen HJC, Grigera JR, Straatsma TP. The missing term in effective pair potentialst. J Phys Chem. 1987;91(24):6269-6271. doi:10.1021/j100308a038

28. Bussi G, Donadio D, Parrinello M. Canonical sampling through velocity rescaling. J Chem Phys. 2007;126(1):014101. doi:10.1063/ 1.2408420

29. Essmann U, Perera L, Berkowitz ML, Darden T, Lee H, Pedersen LG. A smooth particle mesh Ewald method. J Chem Phys. 1995;103 (19):8577-8593. doi:10.1063/1.470117

30. Zhu YX, Jia HR, Pan GY, Ulrich NW, Chen Z, Wu FG. Development of a light-controlled nanoplatform for direct nuclear delivery of molecular and nanoscale materials. J Am Chem Soc. 2018;140 (11):4062-4070. doi:10.1021/jacs.7b13672

31. Rodea-Palomares I, Petre AL, Boltes K, et al. Application of the combination index (CI)-isobologram equation to study the toxicological interactions of lipid regulators in two aquatic bioluminescent organisms. Water Res. 2010;44(2):427-438. doi:10.1016/j.watres. 2009.07.026

32. De Lisi R, Lazzara G, Milioto S. Temperature-controlled poly(propylene) glycol hydrophobicity on the formation of inclusion complexes with modified cyclodextrins. A DSC and ITC study. Phys Chem Chem Phys. 2011;13(27):12571-12577. doi:10.1039/ c1cp20737g

33. Cai C, Liu M, Yan H, et al. A combined calorimetric, spectroscopic and molecular dynamic simulation study on the inclusion complexation of (E)-piceatannol with hydroxypropyl- $\beta$-cyclodextrin in various alcohol+water cosolvents. J Chem Thermodyn. 2019;132:341-351. doi:10.1016/j.jct.2019.01.009
34. Liu M, Chen A, Wang Y, Wang C, Wang B, Sun D. Improved solubility and stability of 7-hydroxy-4-methylcoumarin at different temperatures and $\mathrm{pH}$ values through complexation with sulfobutyl ether-beta-cyclodextrin. Food Chem. 2015;168:270-275. doi:10.1016/ j.foodchem.2014.07.061.

35. Wang C, Liu M, Yuan L, et al. Calorimetric and spectroscopic studies on temperature- and $\mathrm{pH}$-dependent interactions of stimuli-responsive poly (N-isopropylacrylamide) with piceatannol. J Chem Thermodyn. 2016;98:186-192. doi:10.1016/j.jct.2016.03.022

36. Subramanian S, Ross JBA, Ross PD, Brand L. Investigation of the nature of enzyme-coenzyme interactions in binary and ternary complexes of liver alcohol dehydrogenase with coenzymes, coenzyme analogs, and substrate analogs by ultraviolet absorption and phosphorescence spectroscopy. Biochemistry. 1981;20(14):4086-4093. doi:10.1021/bi00517a022

37. Zhao Y, Chen F, Pan Y, et al. Nanodrug formed by coassembly of dual anticancer drugs to inhibit cancer cell drug resistance. ACS Appl Mater Interfaces. 2015;7(34):19295-19305. doi:10.1021/acsami.5b05347

38. Bao H, Zhang Q, Xu H, Yan Z. Effects of nanoparticle size on antitumor activity of 10-hydroxycamptothecin-conjugated gold nanoparticles: in vitro and in vivo studies. Int $J$ Nanomedicine. 2016;11:929-940. doi:10.2147/IJN.S96422

39. Xiao K, Li Y, Luo J, et al. The effect of surface charge on in vivo biodistribution of PEG-oligocholic acid based micellar nanoparticles. Biomaterials. 2011;32(13):3435-3446. doi:10.1016/j. biomaterials.2011.01.021

40. Chauhan VP, Popovic Z, Chen O, et al. Fluorescent nanorods and nanospheres for real-time in vivo probing of nanoparticle shapedependent tumor penetration. Angew Chem Int Ed. 2011;50 (48):11417-11420. doi:10.1002/anie.201104449

41. Gratton SEA, Ropp PA, Pohlhaus PD, et al. The effect of particle design on cellular internalization pathways. Proc Natl Acad Sci USA. 2008;105(33):11613-11618. doi:10.1073/pnas.0801763105

42. Du Y, Li Z, Liu Y, Yang Y, Wang L. Nickel-iron phosphides nanorods derived from bimetallic-organic frameworks for hydrogen evolution reaction. Appl Surf Sci. 2018;457:1081-1086. doi:10.1016/j. apsusc.2018.06.167

43. Yang S, You Q, Yang L, et al. Rodlike MSN@Au nanohybridmodified supermolecular photosensitizer for NIRF/MSOT/CT/MR quadmodal imaging-guided photothermal/photodynamic cancer therapy. ACS Appl Mater Interfaces. 2019;11(7):6777-6788. doi:10.1021/acsami.8b19565

44. Han K, Zhang J, Zhang W, et al. Tumor-triggered geometrical shape switch of chimeric peptide for enhanced in vivo tumor internalization and photodynamic therapy. ACS Nano. 2017;11(3):3178-3188. doi:10.1021/acsnano.7b00216

45. Liu K, Xing R, Zou Q, Ma G, Mohwald H, Yan X. Simple peptide-tuned self-assembly of photosensitizers towards anticancer photodynamic therapy. Angew Chem Int Ed. 2016;55(9):3036-3039. doi:10.1002/ anie. 201509810

46. Guo Y, Jiang K, Shen Z, et al. A small molecule nanodrug by selfassembly of dual anticancer drugs and photosensitizer for synergistic near-infrared cancer theranostics. ACS Appl Mater Interfaces. 2017;9 (50):43508-43519. doi:10.1021/acsami.7b14755

47. Zhang Y, Yang X, Lu Z, Li H, Guo X, Hou W. Self-assembly behavior of amphiphilic poly(ethylene glycol)-conjugated 10-hydroxycamptothecin in water and its cytotoxicity assay. Appl Surf Sci. 2018;459:749-759. doi:10.1016/j.apsusc.2018.08.082

48. Li Q, Liu C, Zhao X, et al. Preparation, characterization and targeting of micronized 10-hydroxycamptothecin-loaded folate-conjugated human serum albumin nanoparticles to cancer cells. Int $J$ Nanomedicine. 2011;6:397-405. doi:10.2147/IJN.S16144

49. Zhang C, Ding Y, Yu LL, Ping Q. Polymeric micelle systems of hydroxycamptothecin based on amphiphilic N-alkyl-N-trimethyl chitosan derivatives. Colloid Surf B Biointerfaces. 2007;55(2):192-199. doi:10.1016/j.colsurfb.2006.11.031 
50. Yameen B, Choi WI, Vilos C, Swami A, Shi J, Farokhzad OC. Insight into nanoparticle cellular uptake and intracellular targeting. J Control Release. 2014;190:485-499. doi:10.1016/j.jconrel.2014.06.038

51. Huang P, Lin J, Wang X, et al. Light-triggered theranostics based on photosensitizer-conjugated carbon dots for simultaneous enhancedfluorescence imaging and photodynamic therapy. Adv Mater. 2012;24 (37):5104-5110. doi:10.1002/adma.201200650

52. Eruslanov E, Kusmartsev S. Identification of ROS using oxidized DCFDA and flow-cytometry. Methods Mol Biol. 2010;594:57-72. doi:10.1007/978-1-60761-411-1 4

53. Yang Z, Li H, Zeng Y, et al. Photosensitizer-loaded branched polyethylenimine-PEGylated ceria nanoparticles for imaging-guided synchronous photo-chemotherapy. ACS Appl Mater Interfaces. 2015;7 (43):24218-24228. doi:10.1021/acsami.5b07702

54. Zhang D, Wu M, Zeng Y, et al. Chlorin e6 conjugated poly(dopamine) nanospheres as PDT/PTT dual-modal therapeutic agents for enhanced cancer therapy. ACS Appl Mater Interfaces. 2015;7 (15):8176-8187. doi:10.1021/acsami.5b01027
55. Hu D, Sheng Z, Gao G, et al. Activatable albumin-photosensitizer nanoassemblies for triple-modal imaging and thermal-modulated photodynamic therapy of cancer. Biomaterials. 2016;93:10-19. doi:10.1016/j.biomaterials.2016.03.037

56. Guo Y, Zhao Y, Wang T, et al. Honokiol nanoparticles stabilized by oligoethylene glycols codendrimer: in vitro and in vivo investigations. J Mater Chem B. 2017;5(4):697-706. doi:10.1039/C6TB02416E

57. Wicki A, Witzigmann D, Balasubramanian V, Huwyler J. Nanomedicine in cancer therapy: challenges, opportunities, and clinical applications. J Control Release. 2015;200:138-157. doi:10.1016/ j.jconrel.2014.12.030

58. Ezeja MI, Anaga AO, Asuzu IU. Acute and sub-chronic toxicity profile of methanol leaf extract of Gouania longipetala in rats. $J$ Ethnopharmacol. 2014;151(3):1155-1164. doi:10.1016/j.jep.2013. 12.034
International Journal of Nanomedicine

\section{Publish your work in this journal}

The International Journal of Nanomedicine is an international, peerreviewed journal focusing on the application of nanotechnology in diagnostics, therapeutics, and drug delivery systems throughout the biomedical field. This journal is indexed on PubMed Central, MedLine, CAS, SciSearch ${ }^{\mathbb{R}}$, Current Contents ${ }^{\mathbb{R}} /$ Clinical Medicine,
Dovepress

Journal Citation Reports/Science Edition, EMBase, Scopus and the Elsevier Bibliographic databases. The manuscript management system is completely online and includes a very quick and fair peer-review

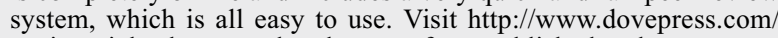
testimonials.php to read real quotes from published authors. 